\title{
Cullin5 deficiency promotes small-cell lung cancer metastasis by stabilizing integrin $\beta 1$
}

\author{
Gaoxiang Zhao,, Liyan Gong, ${ }^{1}$ Dan Su, ${ }^{2}$ Yujuan Jin, ${ }^{1}$ Chenchen Guo, ${ }^{1}$ Meiting Yue, ${ }^{1}$ Shun Yao, ${ }^{1}$ Zhen Qin, ${ }^{1}$ Yi Ye, ${ }^{1,3}$ Ying Tang, ${ }^{1}$ \\ Qibiao Wu, Jian Zhang, ${ }^{1}$ Binghai Cui, ${ }^{1}$ Qiurong Ding, ${ }^{4}$ Hsinyi Huang, ${ }^{1}$ Liang Hu, ${ }^{1}$ Yuting Chen, ${ }^{1,3}$ Peiyuan Zhang, ${ }^{5}$ \\ Guohong Hu, ${ }^{5}$ Luonan Chen, ${ }^{1,3}$ Kwok-Kin Wong, ${ }^{6}$ Daming Gao, ${ }^{1}$ and Hongbin ji ${ }^{1,3}$
}

\begin{abstract}
'State Key Laboratory of Cell Biology, Innovation Center for Cell Signaling Network, CAS Center for Excellence in Molecular Cell Science, Shanghai Institute of Biochemistry and Cell Biology, Chinese Academy of Sciences; University of Chinese Academy of Sciences, Shanghai, China. ${ }^{2}$ Department of Pathology, Zhejiang Cancer Hospital, Hangzhou, Zhejiang, China. ${ }^{3}$ School of Life Science and Technology, Shanghai Tech University, Shanghai, China. ${ }^{4}$ CAS Key Laboratory of Nutrition, Metabolism and Food Safety, Shanghai Institute of Nutrition and Health, Shanghai Institutes for Biological Sciences, Chinese Academy of Sciences, Shanghai, China. ${ }^{5}$ The Key Laboratory of Stem Cell Biology, Institute of Health Sciences, Shanghai Institutes for Biological Sciences, Chinese Academy of Sciences, Shanghai, China. ${ }^{6}$ Laura and Isaac Perlmutter Cancer Center, NYU Langone Medical Center, New York, New York, USA.
\end{abstract}

\begin{abstract}
Metastasis is the dominant cause of patient death in small-cell lung cancer (SCLC), and a better understanding of the molecular mechanisms underlying SCLC metastasis may potentially improve clinical treatment. Through genome-scale screening for key regulators of mouse $\mathrm{Rb}^{-1-} \mathrm{Trp} 53^{-/-}$SCLC metastasis using the pooled CRISPR/Cas9 library, we identified Cullin5 (CUL5) and suppressor of cytokine signaling 3 (SOCS3), two components of the Cullin-RING E3 ubiquitin ligase complex, as top candidates. Mechanistically, the deficiency of CUL5 or SOCS3 disrupted the functional formation of the E3 ligase complex and prevented the degradation of integrin $\beta 1$, which stabilized integrin $\beta 1$ and activated downstream focal adhesion kinase/SRC (FAK/SRC) signaling and eventually drove SCLC metastasis. Low expression levels of CUL5 and SOCS3 were significantly associated with high integrin $\beta 1$ levels and poor prognosis in a large cohort of 128 clinical patients with SCLC. Moreover, the CUL5-deficient SCLCs were vulnerable to the treatment of the FDA-approved SRC inhibitor dasatinib. Collectively, this work identifies the essential role of CUL5- and SOCS3-mediated integrin $\beta 1$ turnover in controlling SCLC metastasis, which might have therapeutic implications.
\end{abstract}

\section{Introduction}

Small-cell lung cancer (SCLC) is the most aggressive and highly metastatic subtype of lung cancer and accounts for approximately $15 \%$ of all lung cancer cases (1). The 5 -year survival rate is only about $10 \%$ for patients with limited-stage SCLC and less than 1\% for those with extensive-stage disease $(2,3)$. Unfortunately, our understanding of SCLC pathogenesis is still very limited, largely because of the lack of clinical samples, e.g., approximately $70 \%$ of patients with SCLC have metastases at the time of initial diagnosis and rarely undergo surgery $(1,4)$. Interestingly, genetic analyses of clinically available SCLC specimens demonstrate that the retinoblastoma protein (RB1) and tumor protein p53 (TP53) alleles are concurrently inactivated in almost all SCLCs (5). Mirroring this clinical observation, homozygous deletion of both $R b 1$ and Trp53 alleles in mouse lung epithelia leads to the formation of SCLC, which pathologically recapitulates the malignant progression of human SCLC (6). This $R b 1^{f / f l} \operatorname{Tr} p 53^{f / f l}$ (referred to herein as RT) SCLC mouse model was proven important for gaining insight into the malignant progression of SCLC. For example, these mouse $R T$

Authorship note: GZ, LG, and DS contributed equally to this work. Conflict of interest: The authors have declared that no conflict of interest exists. License: Copyright 2019, American Society for Clinical Investigation.

Submitted: June 11, 2018; Accepted: November 30, 2018.

Reference information: J Clin Invest. 2019;129(3):972-987.

https://doi.org/10.1172/JCI122779.
SCLCs display strong intratumoral heterogeneity, with different subpopulations containing low metastatic potential, and the cooperation of these tumors is necessary for promoting SCLC metastasis (7). Other studies have also uncovered the important role of epigenetic regulators such as nuclear factor I B (NFIB) and enhancer of zeste 2 polycomb repressive complex 2 subunit (EZH2) in SCLC propagation and metastasis $(8,9)$. Like human SCLC, mouse RT SCLC features the expression of neuroendocrine markers such as neural cell adhesion molecule (NCAM) (6). Moreover, the genetic or molecular alterations frequently observed in human SCLC, such as activation of MYC, SRY-box 2 (SOX2), and other signaling pathways including Notch, Hedgehog, and WNT, are also detectable in mouse RT SCLC (10-16).

Previous studies have indicated the potential involvement of integrins in SCLC malignant progression $(17,18)$. Integrins, importantly, mediate cell-cell adhesion, cell-matrix interactions, as well as cancer cell migration and metastasis $(19,20)$. Integrins are composed of noncovalently associated $\alpha$ and $\beta$ subunits, which form heterodimeric receptor complexes for extracellular matrix (ECM) molecules, with each subunit having a large extracellular domain, a single-membrane-spanning domain, and a short, noncatalytic cytoplasmic tail (19). By directly binding to the ECM components and providing the traction necessary for cell motility and invasion, integrins play the major role in regulating cell proliferation and motility and, as a consequence, metastatic capability. Upon ligation to the ECM, integrins cluster in the 
plane of the membrane and recruit various proteins to form structures known as focal adhesions (21). Despite the lack of kinase activities, integrins can form a cluster and allow the intracellular domain of their $\beta$ subunit to recruit and activate kinases, such as focal adhesion kinases (FAKs), SRC family kinases (SFKs), and other signaling proteins, which then elicit specific intracellular signaling events in response to various environmental stimuli (22). In SCLC, integrin $\beta 1$ is the predominant integrin $\beta$ subunit and known as a potential marker of poor prognosis (17, 18, 23-25). Functionally, integrin $\beta 1$ may facilitate SCLC development via promotion of cell migration and invasion through the formation of various $\alpha 2 \beta 1, \alpha 3 \beta 1, \alpha 6 \beta 1$, and $\alpha v \beta 1$ integrins $(26,27)$. Therefore, integrin $\beta 1$ is considered a potential oncoprotein in the promotion of SCLC malignant progression. However, little is known about how integrin $\beta 1$ is pathologically deregulated in SCLC.

The ubiquitin-proteasome system is important for homeostasis of many key proteins including various oncoproteins and tumor suppressors $(28,29)$. Ubiquitin molecules are conjugated to protein substrates as signals for proteasome degradation. The specificity of to-be-degraded substrates is determined by ubiquitin E3 ligases, which simultaneously associate with specific substrates and position the E2 for ubiquitin conjugation to the substrate (30). Cullin-RING ubiquitin-protein ligases (CRLs) are the largest class of ubiquitin E3 ligases, and Cullin proteins serve as the scaffold and central component of the whole E3 ligase complex by recruiting substrate recognition subunits at the N-terminus and RING proteins (RBX1 and RNF7) at the C-terminus, respectively $(28,31)$. The best-characterized mammalian Cullin family member is Cullin1, which is a component of the multiprotein ubiquitin ligase complex referred to as Skp1-Cul1-F box protein (SCF), or CRL1, and is involved in the degradation of key factors such as c-Myc, $\beta$-catenin, and p 27 (32-34). Different from Cullin1, Cullin5 (CUL5) associates with SOCS box proteins, the RING finger protein RNF7, and the adaptor complex Elongin BC to form functional CRL5 E3 ligases (35). The SOCS box proteins are known to determine the substrate specificity and functions of CRL5 E3 ligases $(36,37)$, and more than 40 SOCS box proteins have been identified (38). Although several substrates of CRL5 have been identified recently (39-41), little is known about how CRL5 E3 ligases function in SCLC.

Recently, the genome-scale CRISPR/Cas9 screen has been proven to be a powerful method for identifying key regulators involved in the malignant progression of cancer, providing a better understanding of disease progression and improved clinical treatments (42). Here, using a mouse RT SCLC spontaneous metastasis model, we performed a genome-wide CRISPR/Cas9 library screen and identified Cul5 as the essential gene in controlling SCLC metastasis. Mechanistically, genetic deletion of Cul5 or Socs 3 substantially enhanced SCLC growth and metastasis through blockage of integrin $\beta 1$ degradation. We found that low expression of CUL5 and SOCS3 was markedly associated with high integrin $\beta 1$ expression in clinical specimens and poor patient survival. Furthermore, treatment with the SRC inhibitor dasatinib dramatically inhibited the metastasis promoted by elevated integrin $\beta 1 /$ FAK/SRC signaling in CUL5-defective SCLC. Taken together, our study reveals the key mechanism governing integrin $\beta 1$ levels in malignant progression of SCLC and identifies SRC as a potential therapeutic target for CUL5-defective SCLC.

\section{Results}

Genome-wide CRISPR/Cas9 library screening identifies candidate genes involved in SCLC metastasis. A previous study has shown that mouse RT SCLC cells frequently harbored low metastatic potential in immunodeficient nude mice allograft assays (6). To screen for genes involved in SCLC metastasis, we infected mouse primary $R T$ SCLC cells with a genome-scale library of lentivirus containing 67,405 sgRNAs targeting 20,611 protein-coding genes and 1,175 miRNA precursors in the mouse genome (43) (Figure 1A and Supplemental Figure 1A; supplemental material available online with this article; https://doi.org/10.1172/JCI122779DS1). After in vitro culturing for 1 week, a total of $6 \times 10^{7}$ cells were subcutaneously injected into the flanks of 60 immunocompromised nude mice $\left(1 \times 10^{6}\right.$ cells per mouse), and spontaneous distant organ metastases were then analyzed 7 weeks after inoculation (Figure 1A). Control (sgCtrl) and library sgRNA-transduced cells formed tumors with NCAM expression (the SCLC biomarker), and these allograft tumors grew at comparable rates and with proliferative indexes (Figure 1, B and C, and Supplemental Figure 1B). Interestingly, in the mice transplanted with library sgRNA-transduced RT cells, SCLC metastases were visually detectable in the lung, liver, chest wall, and diaphragm (Figure 1D). In contrast, none (0 of 5) of the control mice developed detectable metastases in the lungs or liver (Figure 1, D and E). Following the dissection and collection of 461 metastatic tumors for PCR amplification and sequencing, we identified a total of 142 candidate genes potentially involved in SCLC metastasis (Supplemental Table 1). Nf2, a well-known tumor suppressor gene, was among the top-10 candidates (44) (Figure 1F). Importantly, we found that the Cul5 gene stood out as the top hit in the screening and that knockout of $\mathrm{Cul5}$ also resulted in the most diverse spectrum of organ metastases (Figure 1F). These data suggest that Cul5 might play an important role in regulating SCLC metastasis.

Genetic deletion of Cul5 promotes SCLC metastasis. It is worth noting that a component of the CUL5-containing complex, SOCS3, was also among the top hits. CUL5 and SOCS3 are known to form a functional CRL5 E3 ubiquitin ligase complex via the bridging of Elongin BC $(36,45,46)$ to promote substrate degradation (39). We therefore chose to focus on CUL5 and SOCS3 and made individual knockouts to test the impact of their deletion on SCLC metastasis (Figure 2A). We first confirmed the knockout efficiency of Cul5 or Socs 3 sgRNA in vitro (Figure 2B and Supplemental Figure 2A) and then transplanted these cells into nude mice. Distant metastases were assessed 5 weeks after transplantation (Figure 2A). All the mice (5 of 5) from the sgCul5 group developed lung metastases, in stark contrast to no metastases ( 0 of 5 ) in mice in the control group (Figure 2, C and D). Knockout of the Cul5 gene also promoted liver metastasis (1 of 5 mice) and chest wall metastasis (1 of 5 mice) (Figure 2E). Similarly, obvious lung metastases (5 of 5) and lymph node (LN) metastases ( 1 of 5 ) were detected in the sgSocs 3 group (Figure 2, C, D, and F). These data highlight the contribution of both CUL5 and SOCS3 to SCLC metastasis.

Another notable finding is that the allograft tumors from the sgCul5 group grew significantly faster (Supplemental Figure 2B). Although no obvious difference in cell proliferation was detected in vitro (Supplemental Figure 2C), we found that Cul5 knockout clearly promoted anchorage-independent growth of RT cells (Fig- 
A

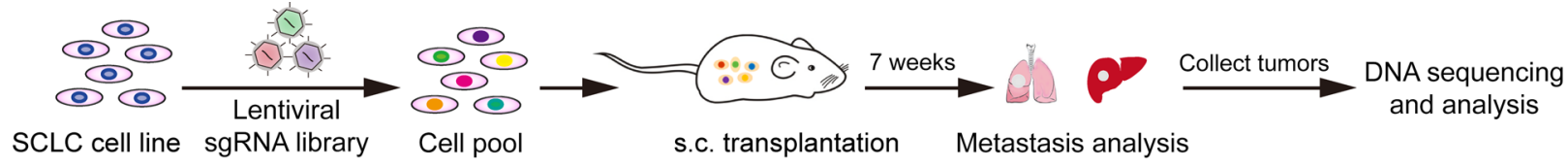

B
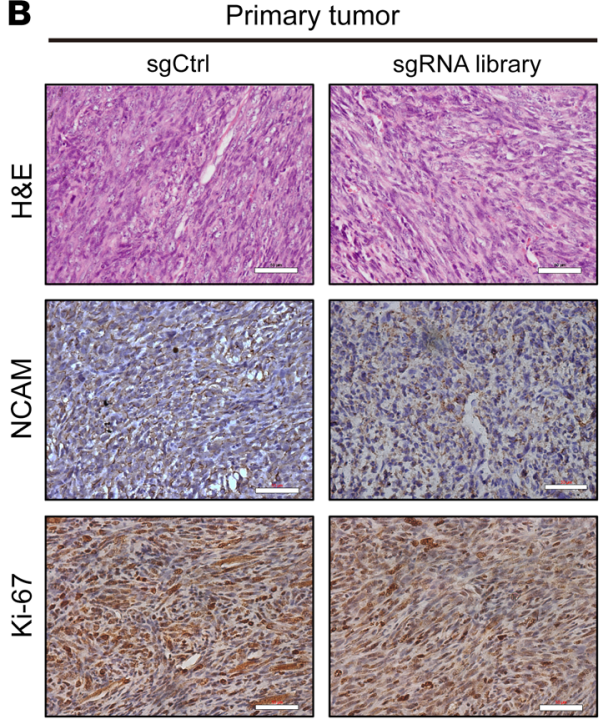

C

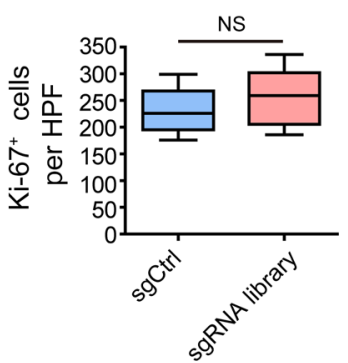
sgRNA library
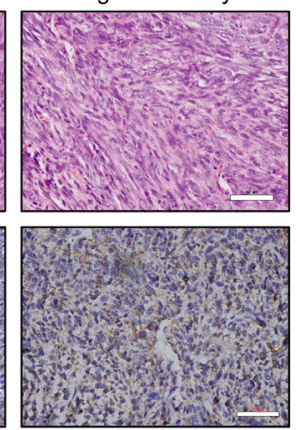

D

\section{:}

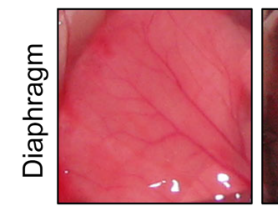

Metastatic tumor
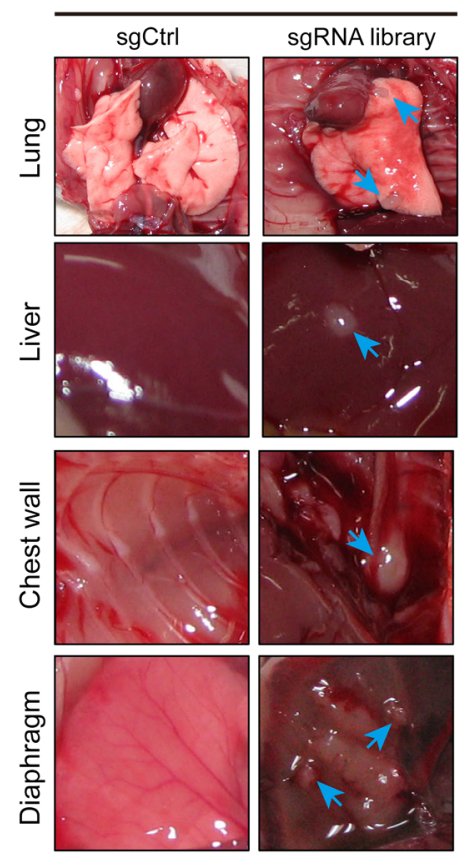

E

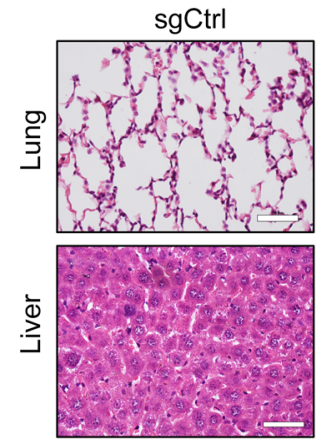

sgRNA library
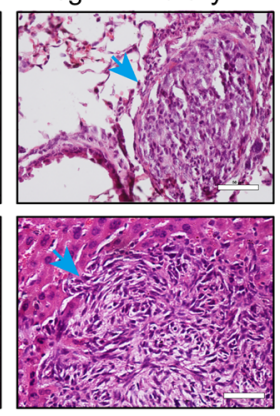

$\overline{\bar{N}}$
3
$\frac{1}{0}$
$\frac{1}{U}$

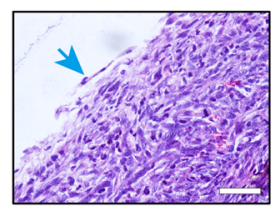

$\frac{E}{0}$
$\frac{\pi}{0}$
$\frac{2}{0}$
$\frac{0}{0}$

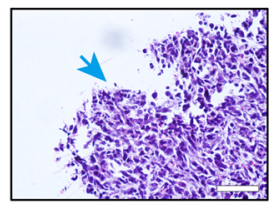

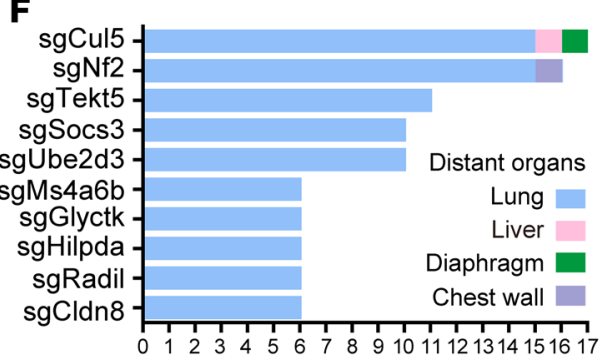

No. of metastatic tumors

Figure 1. In vivo genome-scale CRISPR/Cas9 screening identified potential tumor suppressor genes involved in SCLC metastasis using the mGeCKOa library. (A) Schematic of the genome-scale screen using the mouse CRISPR/Cas9-knockout library (mGeCKOa) in a mouse $R T\left(R b 1^{-/-} \operatorname{Trp} 53^{-/-}\right.$) SCLC allograft assay. (B) Representative H\&E, NCAM, and Ki-67 immunostaining of primary tumors from nude mice subcutaneously transplanted with mouse RT SCLC cells transduced with tomato control sgRNA (sgCtrl) $(n=5)$ or the mGeCKOa lentiviral library (sgRNA library) $(n=60)$. Scale bars: $50 \mu m$. (C) Statistical analysis of the Ki-67+ staining results for primary allograft tumors from the sgCtrl and sgRNA library groups. Data represent the mean $\pm \mathrm{SEM}$. Statistical analysis was performed using Student's $t$ test. (D) Representative photos of the lung, liver, chest wall, and diaphragm from mice transplanted with RT cells transduced with sgCtrl or the sgRNA library. Metastatic tumors are indicated by blue arrows. (E) Representative H\&E staining of tissue from various organs of nude mice subcutaneously transplanted with RT cells transduced with sgCtrl or sgRNA library. Blue arrows indicated metastatic tumors. Scale bars: $50 \mu \mathrm{m}$. (F) Histogram showing the frequency of metastasis enrichment for the top-10 target genes of sgRNAs as revealed by sequencing assays.

ure 2G). These data supported the idea of an essential role of CUL5 in regulating SCLC tumor growth as well as malignant progression. To functionally dissect the role of Cul5 in SCLC metastasis from that in primary tumor growth, we established a postsurgical metastasis model as previously described (47). To this end, we resected the allograft tumors of comparable volume from mice of both groups and allowed the mice to live for another 4 weeks before metastasis analyses. Interestingly, we detected a clear difference in postsurgical metastasis between the sgCul5 group and the control group: lung metastases were found in all the mice from the sgCul5 group (5 of 5) but not in any of the mice from the control group (0 of 5) (Figure 2H). These data identify a critical role of Cul5 in SCLC metastasis in addition to its role in affecting primary tumor growth.

Circulating tumor cells (CTCs) are the hallmark of tumor metastatic capability (48). To test whether Cul5 knockout increased tumor cell intravasation and/or survival, we established RT cells stably expressing GFP with or without Cul5 knockout for allograft assays and quantified CTCs in the blood of nude mice from different groups (Figure 2I). To avoid the impact of tumor volume on SCLC dissemination, we collected whole blood for flow cytometric 
A

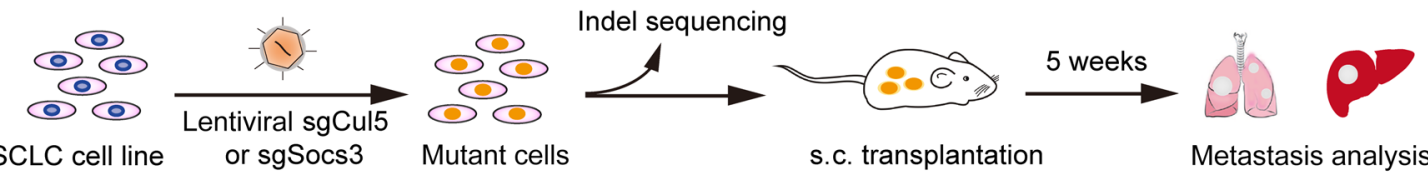

B
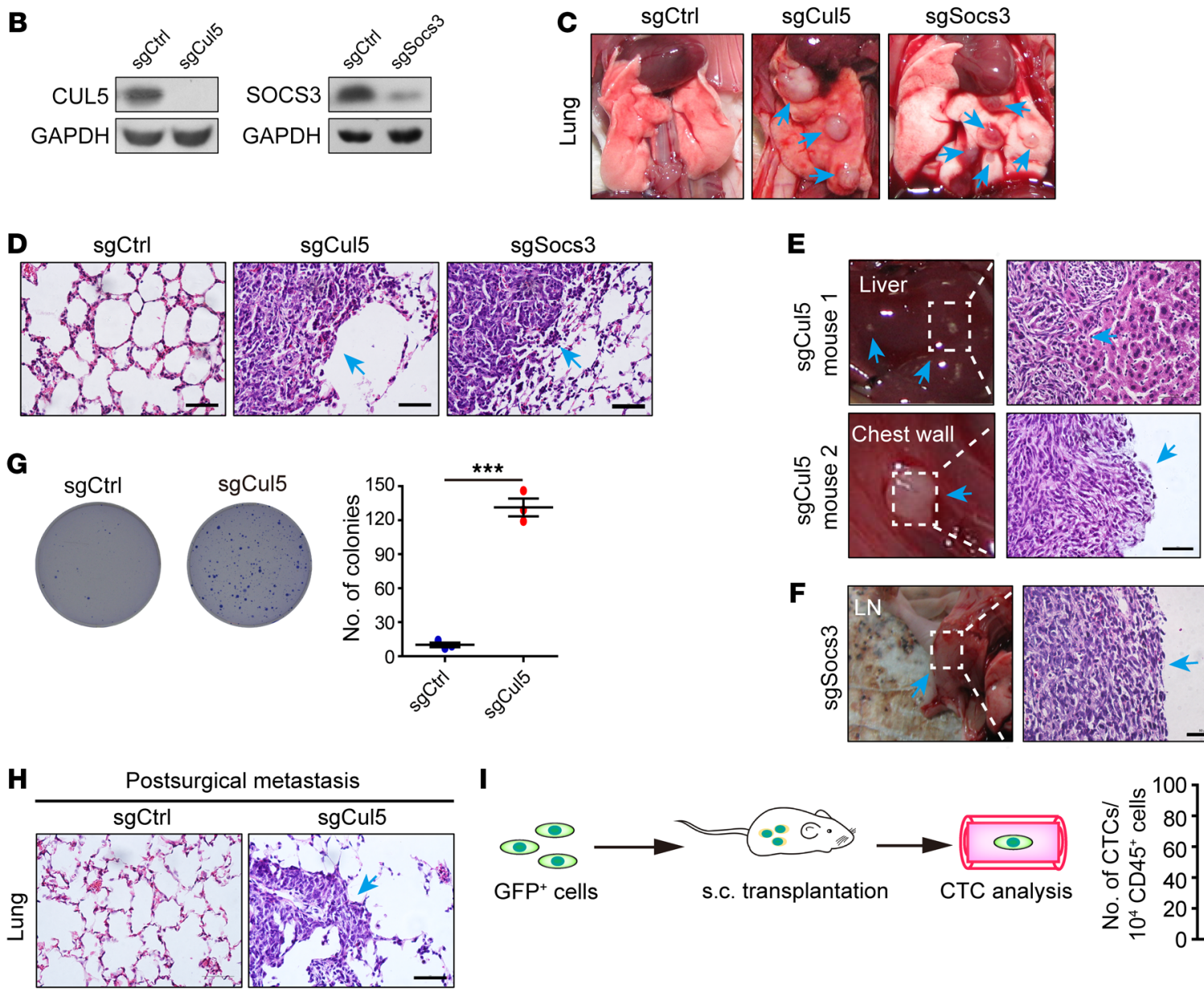

I
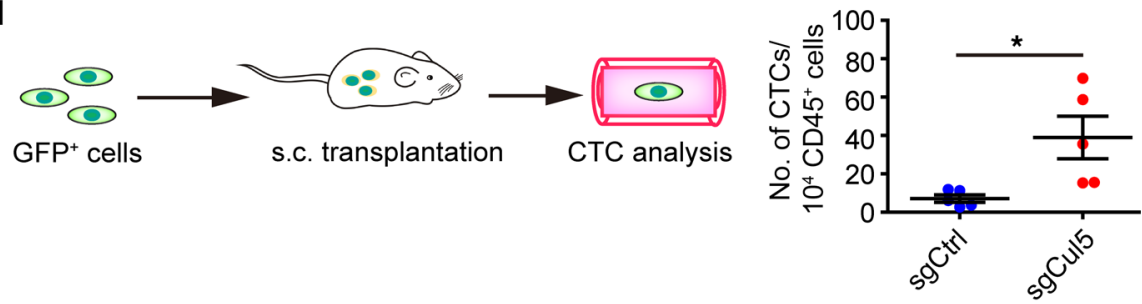

Figure 2. Genetic deletion of Cul5 enhances mouse SCLC metastasis. (A) Diagram of validation of target genes from mGeCKOa screening using individual sgRNAs. (B) Cell lysates from RT cells infected with lentiviral sgCul5 or sgSocs3 vectors were subjected to IB analysis. CAPDH served as a loading control. (C and D) Representative images and H\&E staining of the lung from mice transplanted with RT cells transduced with sgCtrl $(n=5)$, sgCul5 ( $n=5$ ), or sgSocs3 $(n=5)$. Metastatic tumors are indicated by blue arrows. Scale bars: $50 \mu \mathrm{m}$. (E and F) Representative photos and H\&E staining of liver, chest wall, and LN tissue from the different mice transplanted with RT cells transduced with sgCul5 or sgSocs3. Blue arrows indicate metastatic tumors. Scale bar: 50 $\mu \mathrm{m}$. (C) Colony formatiaon capability of RT cells transduced with sgCtrl or sgCul5 in a soft agar assay. Data represent the mean \pm SEM $(n=3)$. ${ }^{* * *} P<0.001$, by Student's $t$ test. (H) Postsurgical metastasis was assessed 4 weeks after resection of comparably sized primary allograft tumors. The surgery was performed 3 weeks after transplantation for the sgCtrl group and 2 weeks after transplantation for the sgCul5 group. Representative $\mathrm{H} \& \mathrm{E}$ staining of lungs is shown. Blue arrow indicates metastasis. Scale bar: $50 \mu \mathrm{m}$. (I) Diagram of CTC analysis. The GFP+ RT cells were transduced with sgCtrl or sgCul5 and then transplanted into the flanks of nude mice. Quantification of circulating $\mathrm{GFP}^{+}$cells per $100 \mu \mathrm{l}$ blood was performed by flow cytometry 4 weeks (sgCtrl) or 3 weeks (sgCul5) after implantation. Dots represent the number of CTCs per 10,000 analyzed blood cells (CD45) for each mouse. Data represent the mean \pm $\operatorname{SEM}\left(n=5\right.$ mice). ${ }^{*} P<0.05$, by Student's $t$ test.

quantification of $\mathrm{GFP}^{+} \mathrm{CTC}$ when mice from both groups developed tumors of comparable volume. We consistently observed more $\mathrm{GFP}^{+}$CTCs in the sgCul5 group than in the control group (Figure 2I). These data indicate that Cul5 deletion promotes SCLC dissemination and metastasis.

The CUL5-SOCS3 ubiquitin ligase complex promotes integrin $\beta 1$ degradation. We next investigated the molecular mechanism of Cul5 deletion in promoting SCLC metastasis. Since deletion of Cul5 or Socs 3 resulted in similar phenotypes for promoting SCLC metastasis, we speculated that CUL5 and SOCS3 might converge upon the same molecule to regulate metastasis. A previous study indicated an essential role of various integrins in SCLC metastasis $(17,18,24,27)$. We postulated that certain integrin molecules might be modulated by the CUL5-SOCS3 E3 ligase complex. We performed a small screen for the substrates of the CUL5-SOCS3 complex and found that knockout of Cul5 and Socs3 led to a dramatic increase in endogenous integrin $\beta 1$ (encoded by ITGB1) but not integrin $\alpha 1$ or $\alpha 2$ (Figure 3, A and B). Consistently, shRNA- 
A

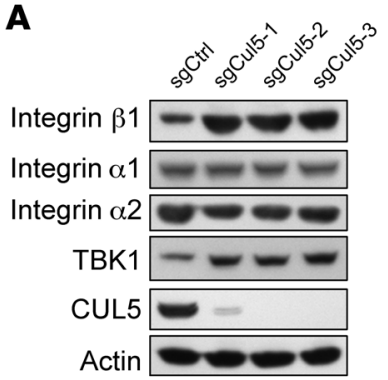

B

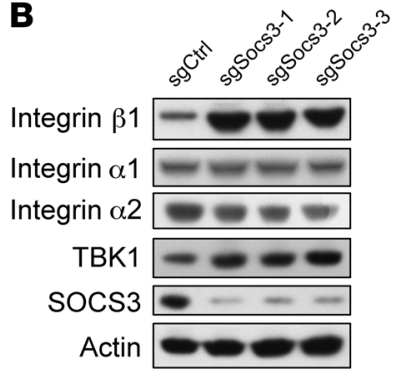

C

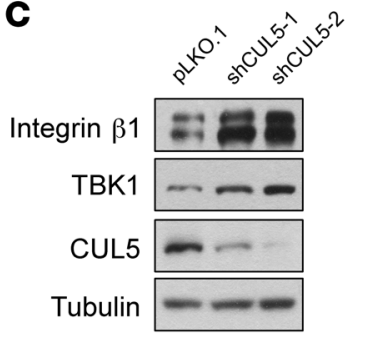

D

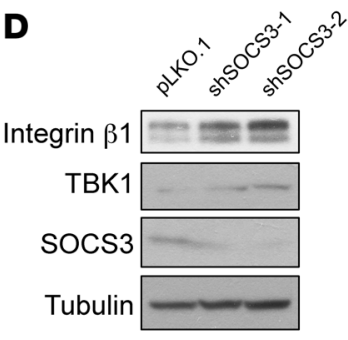

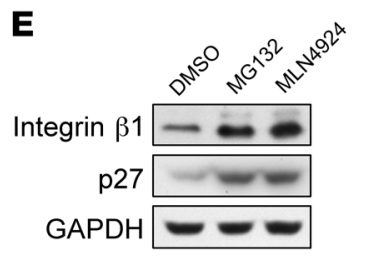
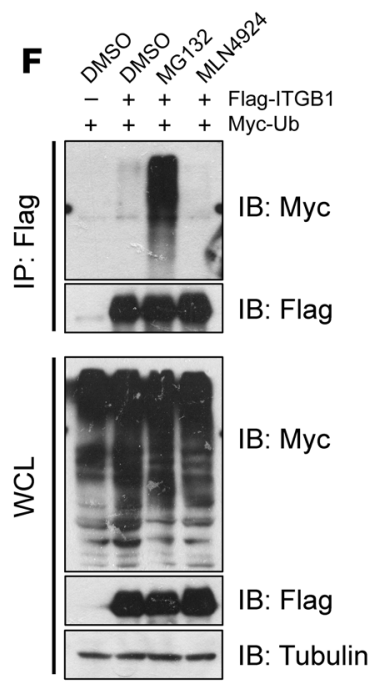

G

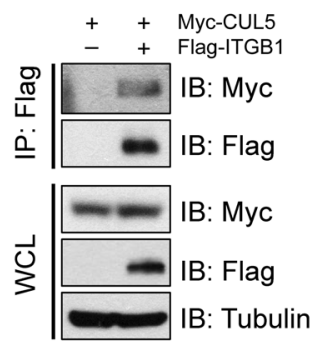

H

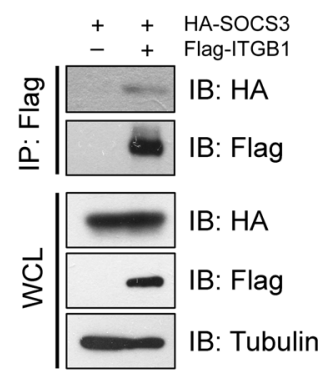

I

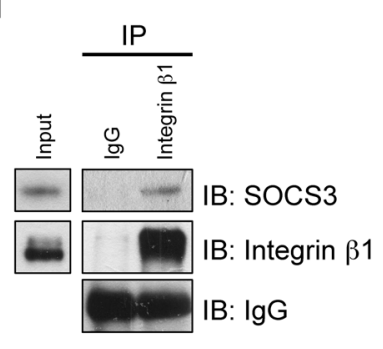

J

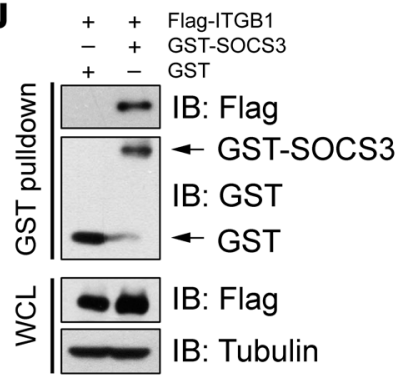

Figure 3. CUL5 and SOCS3 deficiency leads to increased integrin $\boldsymbol{\beta 1}$ protein levels. (A and B) Cell lysates from RT cells infected with the indicated lentiviral sgRNA vectors were subjected to IB analysis. TBK1 was used as a positive control, and actin served as a loading control. (C and D) HeLa cells infected with the indicated shRNA were subjected to IB analysis. TBK1 was used as a positive control, and tubulin served as a loading control. (E) RT cells were treated with $10 \mu \mathrm{M}$ MG132 or $1 \mu \mathrm{M}$ MLN4924 for 10 hours, and the WCLs were subjected to IB analysis. p27 served as a positive control, and GAPDH served as a loading control. (F) HEK293T cells transfected with Flag-ITCB1 and Myc-Ub were treated with DMSO, $10 \mu \mathrm{M}$ MG132, or $1 \mu$ M MLN4924 for 10 hours prior to co-IP and IB analyses. (G and H) HEK293T cells transfected with the indicated plasmids were treated with $10 \mu \mathrm{M}$ MG132 for 10 hours before co-IP and IB analyses. (I) IB analysis of endogenous SOCS3 and integrin $\beta 1$ in HeLa cells, assessed after IP with IgG or anti-integrin $\beta 1$. (J) CST-pulldown analysis of the interaction between CST-SOCS3 and Flag-integrin $\beta 1$.

mediated knockdown of CUL5 and SOCS3 clearly elevated integrin $\beta 1$ protein levels (Figure $3, \mathrm{C}$ and $\mathrm{D}$ ). The mRNA levels of integrin $\beta 1$ in sgCul5- or sgSocs3-transduced cells were comparable to those in control cells (Supplemental Figure 3A), indicating that CUL5 and SOCS3 mainly modulated integrin $\beta 1$ at the posttranscriptional level. Further, treatment with the proteasome inhibitor MG132 or the NEDD8-activating enzyme inhibitor MLN4924 (often used to suppress CRL E3 ligase activity) (49) caused an obvious elevation of integrin $\beta 1$ protein levels, but only MG132 increased its polyubiquitination, indicating that integrin $\beta 1$ is an unstable protein that is likely governed by CRL E3 ligase complexes (Figure 3, E and F, and Supplemental Figure 3B). To further confirm the role of the CUL5-SOCS3 complex in regulating integrin $\beta 1$, we validated the interaction between integrin $\beta 1$ and CUL5-SOCS3 by co-IP and GST-pulldown assays (Figure 3, G-J, and Supplemental Figure 3, C-E). These data support the notion that integrin $\beta 1$ is a bona fide degradation substrate of CUL5-SOCS3 complex.
Unlike other CRLs, CRL5 depends on RNF7 rather than RBX1 to ubiquitinate the substrates (31). Indeed, knockdown of RNF7 but not RBX1 dramatically increased integrin $\beta 1$ protein levels (Figure 4A). We further showed that knockdown of CUL5, SOCS3, and RNF7 clearly extended the half-life of endogenous integrin $\beta 1$ (Figure 4, B-E, and Supplemental Figure 3F), suggesting that CUL5, SOCS3, and RNF7 are the components of the master E3 ligase responsible for integrin $\beta 1$ degradation. K48-, K63-, and K11-linked polyubiquitination is regarded as a classical signal to guide the substrates to the proteasome for degradation (50), while a recent study indicated that the CRL5 E3 ligase may degrade substrates via K11 linkage (39). We consistently found that ectopic SOCS3 promoted K11-only ubiquitin (K11-only Ub) (all K residues mutated to R except K11), but not K11-R-Ub (only K11 mutated to $\mathrm{R}$ ), to mediate integrin $\beta 1$ polyubiquitination (Figure $4 \mathrm{~F}$ and Supplemental Figure 3G). Therefore, the CUL5-SOCS3 ligase complex mainly promotes K11-linked polyubiquitination of integrin $\beta 1$. 
A

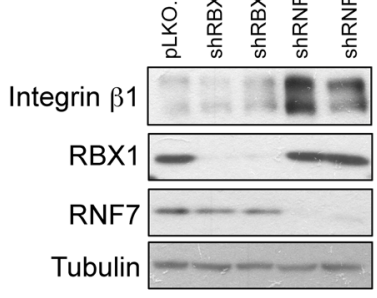

B

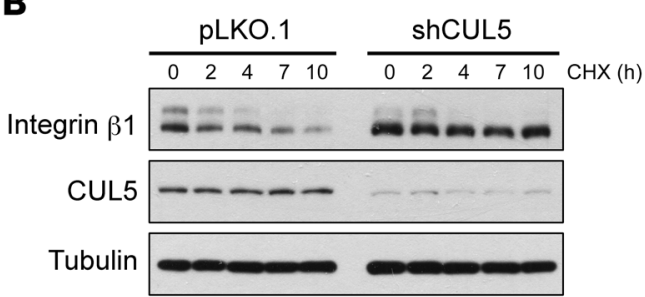

D

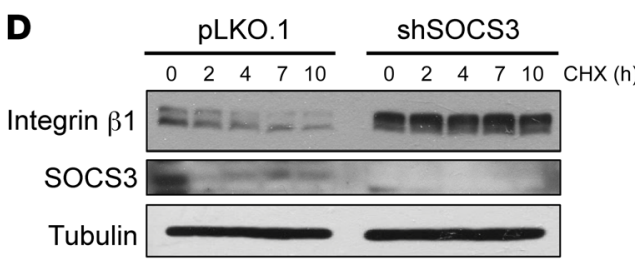

G

$\mathbf{F}$

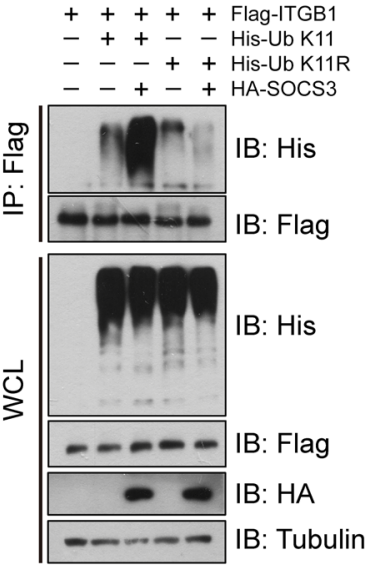

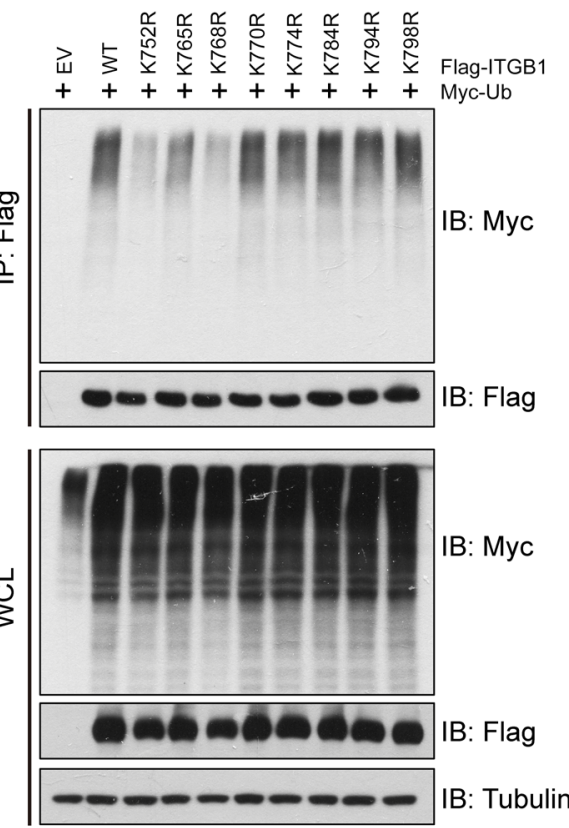
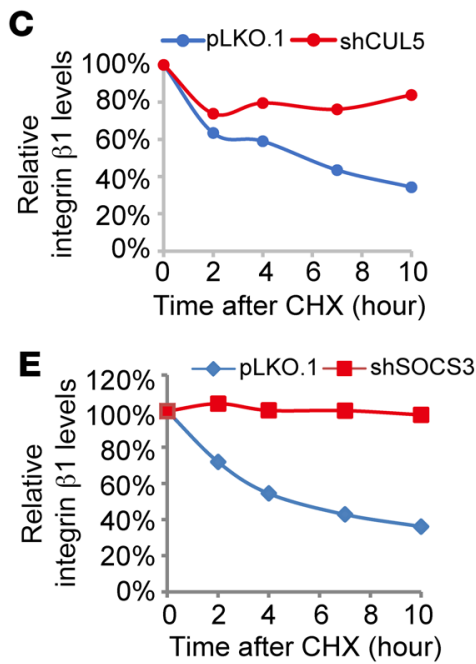

H

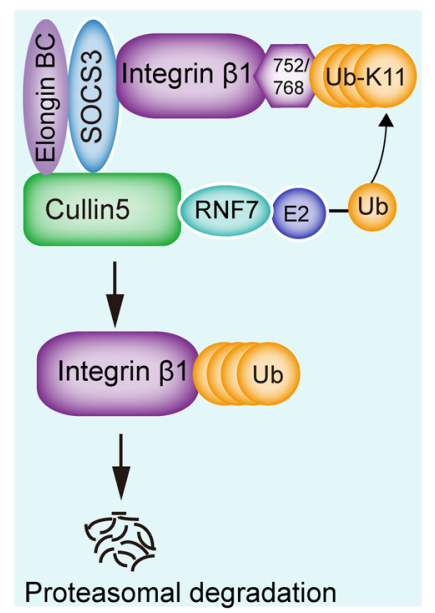

Figure 4. The CUL5-SOCS3 E3 ubiquitin ligase complex promotes integrin $\beta 1$ ubiquitination and degradation. (A) HeLa cells infected with the indicated shRNA were subjected to IB analysis. Tubulin served as a loading control. (B-E) Hela cells transfected with shCUL5 or shSOCS3 were treated with $100 \mu \mathrm{g} / \mathrm{ml} \mathrm{CHX}$ prior to IB analysis. Tubulin served as a loading control. Integrin $\beta 1$ protein levels were quantified by normalization to tubulin. (F) HEK293T cells transfected with Flag-ITCB1, His-tagged Ub (K11 or the K11R mutant), or HA-SOCS3 were treated with $10 \mu M$ MG132 for 10 hours before co-IP and IB analyses. (G) HEK293T cells transfected with WT integrin $\beta 1$ or various lysine-to-arginine (K-R) mutants of integrin $\beta 1$ were treated with $10 \mu \mathrm{M}$ MG132 for 10 hours before co-IP and IB analyses. EV, empty vector. (H) Scheme illustrating how the CUL5-SOCS3 E3 ligase complex results in integrin $\beta 1$ ubiquitination and degradation.

To further identify the specific lysine residues in the cytoplasmic region of integrin $\beta 1$ that are conjugated with the ubiquitination chain, we generated a set of integrin $\beta 1$ mutants by substituting individual lysine (K) residues with arginine (R) residues and tested their responses to CUL5-SOCS3-mediated ubiquitination and degradation (Supplemental Figure 3H). We found that integrin $\beta 1-K 752 \mathrm{R}$ and integrin $\beta 1-\mathrm{K} 768 \mathrm{R}$ mutants were more resistant to SOCS3-induced degradation and less ubiquitinated compared with WT integrin $\beta 1$ (Figure $4 \mathrm{G}$ and Supplemental Figure 3, I-K). As SOCS3-mediated protein degradation may require specific phosphorylation of tyrosine residues (51), we examined whether degradation of integrin $\beta 1$ is also phosphorylation dependent. We found that neither the interaction between SOCS3 and integrin $\beta 1$, nor the degradation of integrin $\beta 1$ was affected by the mutation of tyrosine residues in the cytoplasmic part of integrin $\beta 1$ (Supplemental Figure 3, L and M). Collectively, these data demonstrate that the CUL5-SOCS3 complex specifically recognizes integrin $\beta 1$ and modulates K11-linkage-specific integrin $\beta 1$ degradation (Figure $4 \mathrm{H})$. Indeed, in vivo data from the $R b 1^{f / f l} \operatorname{Tr} p 53^{\text {If/l}}$ mouse model showed that CUL5 and SOCS3 were significantly downregulated, together with integrin $\beta 1$ upregulation, in the metastatic tumors in comparison with primary tumors (Supplemental Figure 3, N-T), further supporting the link between the CUL5-SOCS3 complex and integrin $\beta 1$ in SCLC metastasis. 
Deficiency of the CUL5-SOCS3 complex is associated with increased integrin $\beta 1$ protein levels and poor prognosis for patients with SCLC. Through our analysis of public data sets in the cBioPortal database (http://cbioportal.org), we found that 4 patients bore CUL5 mutations and 1 patient had a SOCS3 mutation among 101 SCLC cases examined (Supplemental Figure 4, A-D). Moreover, the median survival was $40 \%$ shorter in the CUL5 mutation group (15 months vs. 25 months) and 8\% shorter in the SOCS3 mutation group (23 months vs. 25 months) (Supplemental Figure 4, C and D).

As integrin $\beta 1$ abundance is tightly controlled by the CUL5SOCS3 E3 ligase complex, we next investigated whether these patient-derived CUL5 or SOCS3 mutations impaired the complex formation as well as integrin $\beta 1$ degradation. Interestingly, all four CUL5 mutations tested (including K137T, R167I, M262V, and K286R) drastically reduced CUL5 interaction with SOCS3 (Figure 5, A and B). When reexpressed, only WT CUL5, but not any mutants, appeared to reduce endogenous integrin $\beta 1$ levels in sgCUL5-treated SCLC cells (Figure 5C). We also examined the SOCS3 mutation K28R found in human SCLC. Strikingly, we found that the SOCS3 K28R mutant failed to interact with integrin $\beta 1$ and promote its degradation, which is very similar to what occurs with deletion of the substrate-interacting SH2 domain $(37,52)$ (Figure $5, \mathrm{D}-\mathrm{F})$. These results support the idea that the CUL5 and SOCS3 mutations detected in SCLC disable CUL5-SOCS3 E3 ligase complex formation and prevent the degradation of integrin $\beta 1$.

In order to gain a more general insight into the association between the CUL5-SOCS3 complex and integrin $\beta 1$ in clinical specimens, we examined a total of 128 human SCLC samples. We found a significant inverse correlation between integrin $\beta 1$ levels and expression of CUL5 and/or SOCS3 (Figure 5, G-I) and that SCLC specimens from extensive-stage patients were associated with low CUL5 and SOCS3 levels and high integrin $\beta 1$ levels (Figure 5J). We further found that low CUL5 and SOCS3 levels and high integrin $\beta 1$ levels were significantly associated with shorter patient survival (Figure 5, K and L), respectively. Moreover, RNASeq results from 19 human SCLC specimens showed that SOCS3 mRNA levels were significantly reduced in tumor tissues when compared with levels in nontumor tissues (Figure 5M), consistent with previously published data (10) (Supplemental Figure 4E). These results suggest that inactivation of the CUL5-SOCS3 complex caused by mutations or expressional downregulation of CUL5 and/or SOCS3 might impair integrin $\beta 1$ degradation and lead to poor prognosis for patients with SCLC.

Deficiency of the CUL5-SOCS3 complex promotes SCLC metastasis via activation of integrin $\beta 1 / F A K / S R C$ signaling. We continued to explore the mechanisms underlying SCLC metastasis driven by CUL5-SOCS3 deletion and integrin $\beta 1$ stabilization. It is well established that integrins signal through the recruitment and activation of downstream factors including FAK and SRC, which form a dual kinase complex and promote cancer cell migration $(21,53)$. We therefore tested whether the CUL5-SOCS3 deletion affected FAK/SRC signaling through integrin $\beta 1$ in SCLC. As with integrin $\beta 1$ overexpression, the depletion of CUL5 or SOCS3 in human and mouse SCLC cells promoted FAK Tyr397 phosphorylation (p-FAK) and SRC Tyr416 phosphorylation (p-SRC), indicating FAK and SRC activity, respectively, without affecting FAK and SRC protein abundance (Figure 6, A and B, and Supplemental Figure 5B). Ecto- pic expression of SOCS3 consistently led to a drastic decrease in the levels of integrin $\beta 1$ as well as p-FAK and p-SRC (Figure 6C). Moreover, the depletion of integrin $\beta 1$ effectively inhibited the phosphorylation of FAK and SRC triggered by knockout of Cul5 or Socs3 (Figure 6, D and E, and Supplemental Figure 5A). These data demonstrate that deletion of the CUL5-SOCS3 complex activates FAK/SRC pathways via stabilization of integrin $\beta 1$ in SCLC.

Recent studies have shown that the FAK-SRC complex is activated in many types of cancers and necessary for integrin-mediated tumor cell migration and metastasis $(54,55)$. It is possible that the elevated integrin $\beta 1 / F A K / S R C$ signaling promoted SCLC cell migration and metastasis on a CUL5- and SOCS3-defective background. Indeed, ectopic expression of integrin $\beta 1$ activated FAK and SRC kinases and promoted the migration of SCLC cells in Transwell assays (Supplemental Figure 5, B-D). Consistently, deletion of CUL5 or SOCS3 promoted SCLC cell migration, which could be largely abolished by concurrent deletion of integrin $\beta 1$ or FAK and SRC kinases (Figure 6, F and G). Concordantly, overexpression of CUL5 or SOCS3 inhibited SCLC cell migration (Supplemental Figure 5, E-G). These data demonstrate that the CUL5-SOCS3 complex might modulate SCLC cell migration at least partially through governing integrin $\beta 1$ protein abundance and downstream FAK/SRC signaling.

Our data further showed that Cul5- or Socs3-knockout SCLC cells clearly increased the incidence of lung metastases in allograft experiments (Figure 6, $\mathrm{H}-\mathrm{J}$ ), similar to what was observed with integrin $\beta 1$ overexpression (Supplemental Figure $5 \mathrm{H}$ ). Moreover, ectopic expression of degradation-resistant mutant integrin $\beta 1-K 752 \mathrm{R} / \mathrm{K} 768 \mathrm{R}$ promoted SCLC metastasis, even in the case of SOCS3 overexpression (Supplemental Figure 5, I and J). Strikingly, deletion of Itgb1, Fak, or Src almost completely abolished the strong metastasis triggered by Cul5 and Socs 3 deletion (Figure 6, H-J). We also observed increased transcription of classical metastasispromoting genes, including $M m p 14$, Vegf, and Nfib, and reduced anoikis in Cul5- or Socs3-knockout cells (Supplemental Figure 5, K and $\mathrm{L}$ ). Together, these results strongly suggest that disruption of the CUL5-SOCS3 complex activates integrin $\beta 1 / F A K / S R C$ signaling and thereby promotes SCLC metastasis.

Dasatinib treatment inhibits SCLC metastasis driven by CUL5 deficiency. Previous studies have identified an essential role of SRC in malignant cancer progression (56). Given the robust inhibition of Cul5-defective SCLC metastasis through Src deletion, we reasoned that SRC inhibition might work effectively to suppress SCLC metastasis. To this end, we used the FDA-approved SRC inhibitor dasatinib to treat allograft SCLC tumors with or without Cul5 knockout. Tumor-bearing mice were intragastrically administered vehicle or dasatinib. We found that dasatinib treatment had almost no effect on allograft tumors with intact Cul5 (sgCtrl group) (Figure 7A and Supplemental Figure 6, A and B). In contrast, dasatinib treatment significantly suppressed primary tumor growth in the sgCul5 group (Figure 7A). Reduced cell proliferation and increased apoptosis were further confirmed by Ki-67 and cleaved caspase-3 (CC3) staining (Figure 7, B and C). More important, dasatinib treatment drastically reduced the number and burden of lung metastases in the sgCul5 group, without significant body weight loss (Figure 7, D-F, and Supplemental Figure 6, $\mathrm{C}-\mathrm{E})$. As integrin $\beta 1$ is known to promote angiogenesis, by which 
A
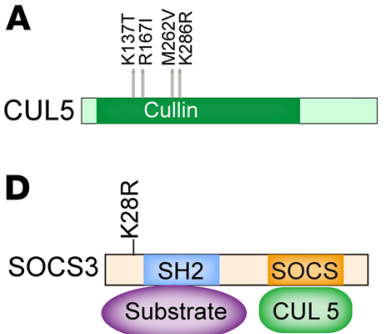

E
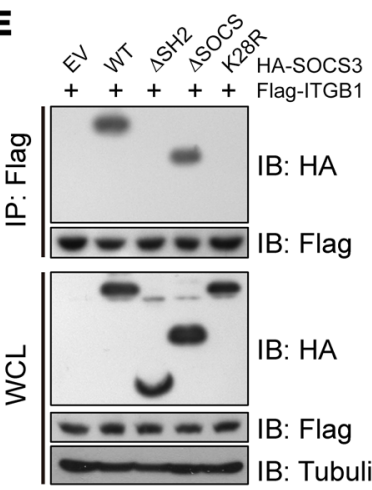

IB: HA

B: Flag

IB: Tubulin
B
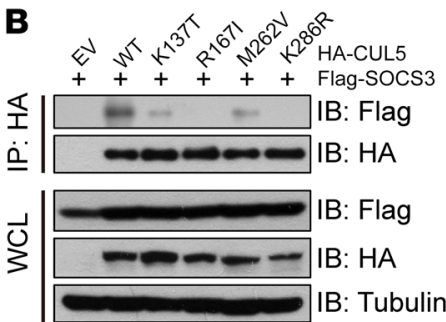

C

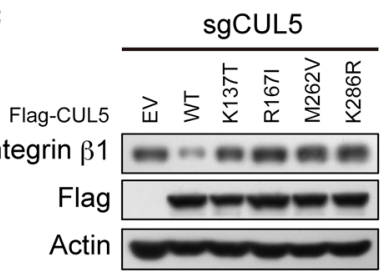

$\mathbf{F}$

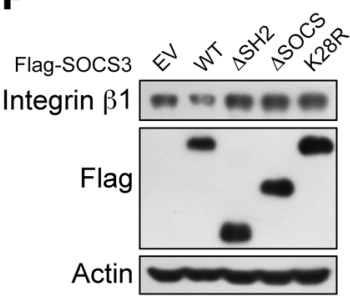

G

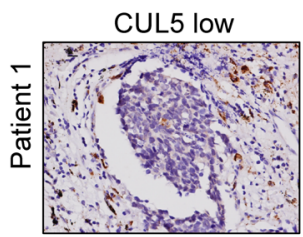

CUL5 high

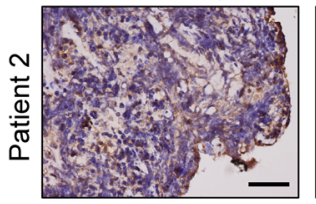

Integrin $\beta 1$ high

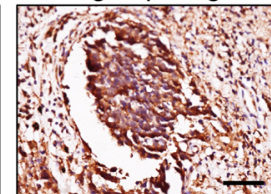

Integrin $\beta 1$ low

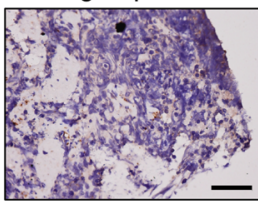

H

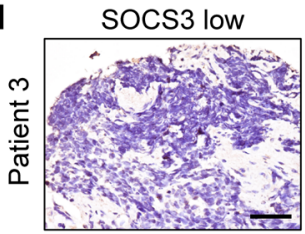

Socs3 high
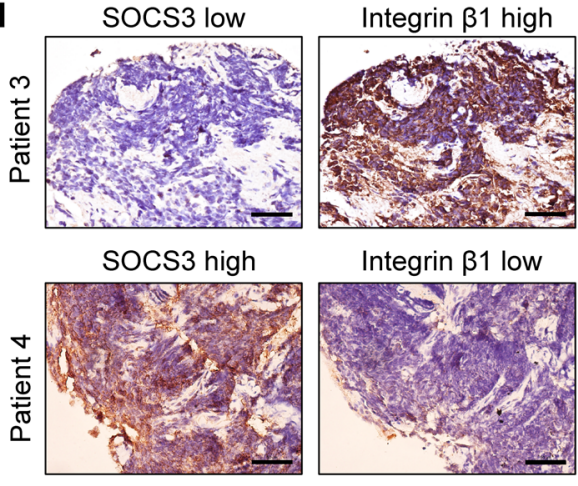

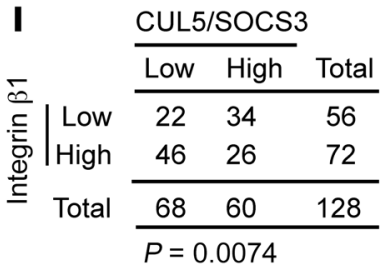

J

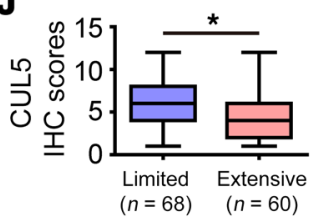

(Stage)

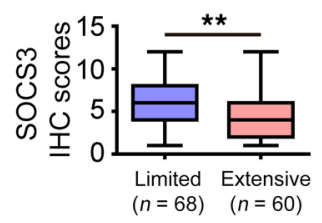

(Stage)

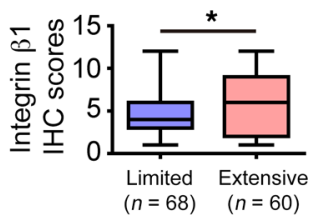

(Stage)
K

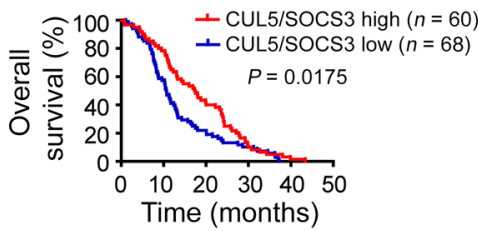

L

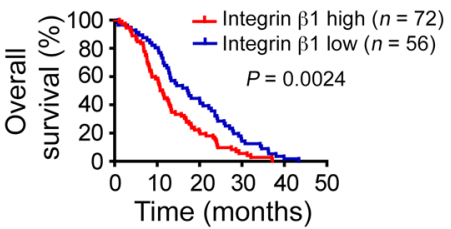

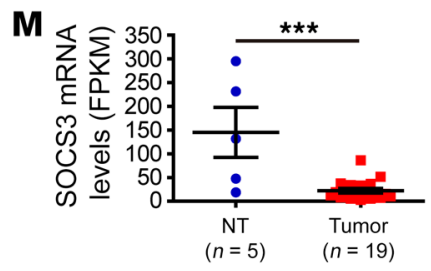

Figure 5. SCLC-associated CUL5 and SOCS3 deficiency promotes SCLC progression by impairing integrin $\beta 1$ degradation. (A) Schematic illustration of CUL5 mutants reported in human SCLC. (B) IB analyses of WCLs and IP from HEK293 cells transfected with SCLC-associated CUL5 mutants and FlagSOCS3. Cells were treated with $10 \mu \mathrm{M}$ MG132 for 10 hours before harvesting. Tubulin was used as a loading control. (C) IB analysis of lysates from the human SCLC cell line H345 stably expressing Flag-tagged WT-CUL5 or the indicated SCLC-associated CUL5 mutants. Actin was used as a loading control. (D) Schematic diagram of SOCS3 domains and a mutation found in human SCLC. (E) Co-IP analysis of the interaction of Flag-ITCB1 with the indicated SOCS3 constructs in HEK293T cells. Tubulin was used as a loading control. (F) IB analysis of lysates from H345 cells transfected with the indicated constructs. Actin was used as a loading control. (G and $\mathbf{H})$ Representative images of IHC staining for integrin $\beta 1$, CUL5, and SOCS3 from 128 SCLC specimens. Scale bars: $50 \mu \mathrm{m}$. (I) A significant negative correlation between CUL5-SOCS3 and integrin $\beta 1$ levels was observed in human SCLC clinical samples. Statistical significance was determined using a $\chi^{2}$ test. CUL5/SOCS3 low indicates that CUL5 and/or SOCS3 levels were low; CUL5/SOCS3 high indicates that both CUL5 and SOCS3 levels were high. (J) Relative CUL5, SOCS3, and integrin $\beta 1$ IHC scores were plotted on the basis of the Wilcoxon matched-pairs test. Data represent the mean \pm SEM. ${ }^{*} P<0.05$ and ${ }^{*} P<0.01$, by Student's $t$ test. (K and $\mathbf{L}$ ) Kaplan-Meier curves show the overall survival of SCLC patients with high or low expression of CUL5 and SOCS3 (K) and integrin $\beta 1$ (L). Statistical significance was determined by log-rank test. (M) Sequencing of mRNA indicated that SOCS3 levels were significantly downregulated in human SCLC compared with levels in nontumor (NT) tissue. ${ }^{* *} P<0.001$, by Student's $t$ test. 
A

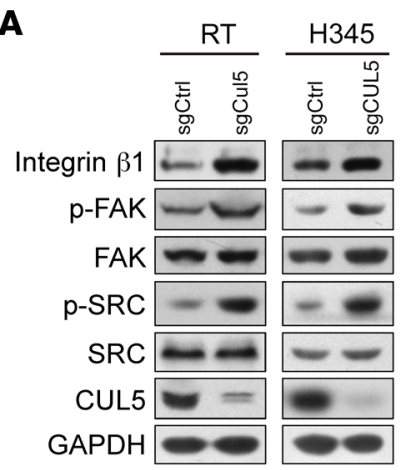

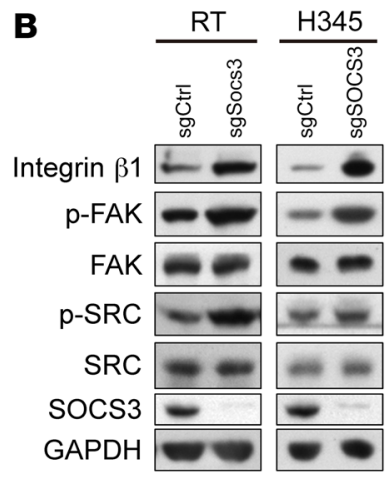

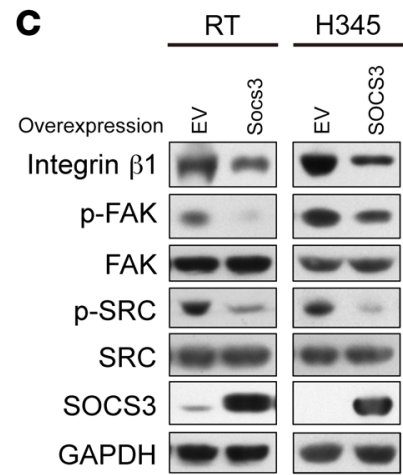

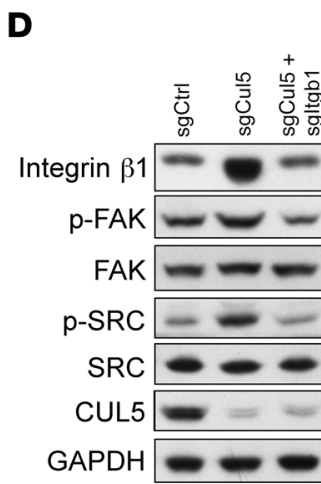
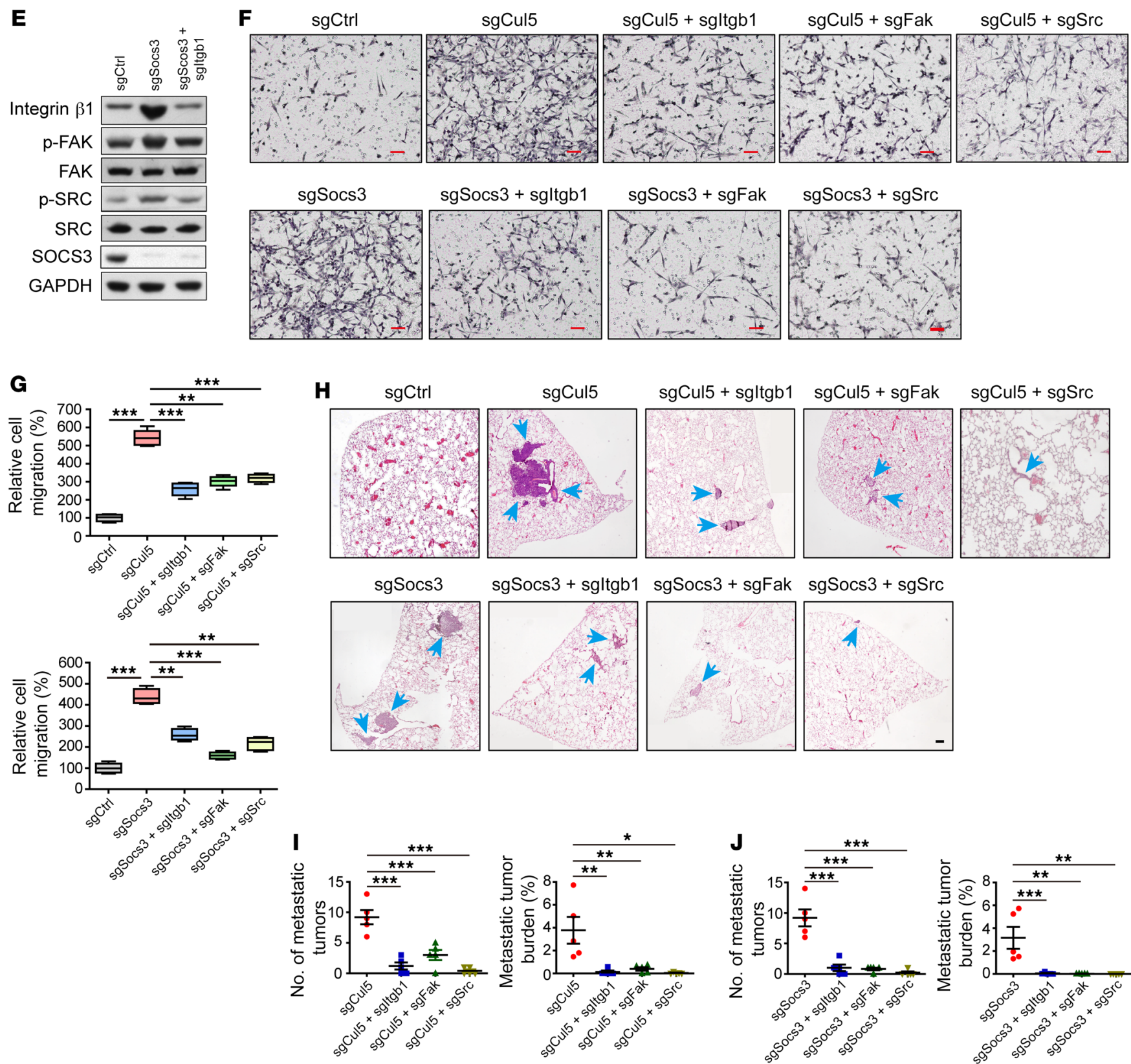
Figure 6. The CUL5-SOCS3 complex inhibits SCLC cell migration and metastasis by attenuating integrin $\beta 1 / F A K / S R C$ signaling. ( $A$ and $B$ ) IB analyses of integrin $\beta 1$ levels, phosphorylation levels of FAK and SRC in RT and H345 cells depleted of (A) Cul5 or (B) Socs3. GAPDH was used as a loading control. (C) IB analyses of integrin $\beta 1$ levels and phosphorylated and total protein levels of FAK and SRC in RT and H345 cells overexpressing SOCS3. GAPDH was used as a loading control. (D) Integrin $\beta 1$ levels and the phosphorylation status of FAK and SRC were detected by IB of RT cells with knockout of Cul5 or knockout of both Cul5 and Itgb1. GAPDH was used as a loading control. (E) Integrin $\beta 1$ levels and phosphorylation status of FAK and SRC were detected by IB in RT cells with knockout of Socs3 or knockout of both Socs3 and Itgb1. GAPDH served as a loading control. (F) Representative images of migrated RT cells treated with indicated sgRNA constructs in a Transwell assay. Double-knockout of sgCul5 or sgSocs3 with sgltgb1, sgFak, or sgSrc was carried out to test the rescue effects. Scale bars: $50 \mu \mathrm{m}$. (G) Quantification of cell migration. Data represent the mean \pm SEM. ${ }^{* *} P<0.01$ and ${ }^{* * *} P<0.001$, by 1 -way ANOVA followed by the Newman-Keuls multiple comparisons test. (H) Representative H\&E staining of lung tumor sections from nude mice transplanted with RT cells transduced with the indicated sgRNAs. Blue arrows indicate metastatic tumors. Scale bar: $50 \mu \mathrm{m}$. (I and J) Statistical analyses of lung tumor numbers and tumor burden for different groups ( 5 mice per group, 5 weeks). Data represent the mean \pm SEM. ${ }^{*} P<0.05,{ }^{* *} P<0.01$, and ${ }^{* * *} P<0.001$, by 1 -way ANOVA followed by the Newman-Keuls multiple comparisons test.

it facilitates cancer cell dissemination and metastasis (57), we assessed the level of the endothelial marker CD31 as the indicator of angiogenesis. We found that dasatinib treatment also significantly reduced CD31 levels in sgCul5 allograft tumors (Figure 7, B and C, and Supplemental Figure 6, A and B). Collectively, these results indicate that CUL5-defective mouse SCLC tumors might be sensitive to dasatinib treatment.

We next examined the effects of dasatinib on human SCLC. We first performed sgRNA-mediated knockout of CUL5 and SOCS3 in multiple human SCLC cell lines including H345, H82, H2O9, and H446. An in vitro cell proliferation assay showed that knockout of CUL5 and SOCS3 had no impact on sensitivity to dasatinib treatment (Supplemental Figure 6, F-H). In contrast, dasatinib treatment significantly inhibited the migration of CUL5- and SOCS3-knockout cells (Figure 8, A and B, and Supplemental Figure 6, I and J). We further subcutaneously transplanted CUL5-knockout human H345 cells into nude mice to establish the xenograft tumors for dasatinib treatment. Importantly, we found that dasatinib treatment significantly inhibited the growth of CUL5-knockout xenograft tumors (Figure 8C), and immunohistochemical staining indicated significantly decreased proliferation and increased apoptosis in CUL5-knockout tumors after treatment with dasatinib (Figure 8, D and E, and Supplemental Figure 6, K and L). These results further suggest that dasatinib treatment might serve as an effective therapy for human CUL5-defective SCLC.

\section{Discussion}

Here we performed genome-wide CRISPR/Cas9-knockout screening to identify important regulators of SCLC metastasis using an allograft tumor model. Our results suggested that deficiency of either CUL5 or SOCS3, two components of the CRL5-E3 complex, will substantially promote SCLC metastasis by stabilizing integrin $\beta 1$ and stimulating integrin $\beta 1$ downstream signaling including FAK and SRC (Figure 8F). Our work further indicates that the SRC inhibitor dasatinib might serve as an effective therapy for SCLC with CUL5 and SOCS3 deficiency (Figure 8F). Together, our study not only uncovers mechanisms underlying SCLC metastasis but also provides a potential therapeutic strategy for the treatment of metastatic SCLC.

As the largest ubiquitin ligase family, CRL complexes are speculated to modulate the proteolysis of thousands of proteins (28). CRL5 complexes were previously shown to degrade signaling proteins such as JAK2 (52), gp130 (58), and Cas (59) and found to be downregulated in various cancers (60-62). However, the potential role of CRL5 in tumor metastasis, especially in SCLC metastasis, remains unexplored. In the present study, we identified CUL5 and its adaptor protein SOCS3 as potential suppressors of SCLC metastasis through the formation of a functional CRL5 E3 complex to degrade integrin $\beta 1$. In keeping with a critical role of the CUL5-SOCS3 complex in SCLC, we found that SOCS3 mRNA levels are decreased in human SCLC specimens and that decreased expression of CUL5 and SOCS3 is associated with a poor prognosis for patients with SCLC. In accordance with this, CUL5 and SOCS3 mutants identified in clinical specimens failed to form a functional E3 ligase complex to degrade integrin $\beta 1$. The patients with CUL5 and SOCS3 mutations also tended to have a poorer prognosis, further supporting the importance of CUL5 and SOCS3 dysfunction in SCLC metastasis. These findings also indicate that deficiency of Cul5 and Socs3, in addition to loss of $R b 1$ and Trp53, would create a more useful de novo cancer model with which to study malignant progression and metastasis of SCLC and would lead to improved therapeutic strategies.

Previous studies have highlighted the heterodimerization between integrin $\beta 1$ and other $\alpha$ forms in cancer metastasis (20, 63). The strategy of targeting integrin $\beta 1$ showed some efficacy in blocking cancer growth and metastasis in preclinical models and in patients with advanced solid tumors (20). In fact, SCLC tumors frequently grow in an ECM-rich environment and are surrounded by ECM proteins such as fibronectin (an integrin $\alpha 5 \beta 1$ ligand), collagen IV (an integrin $\alpha 1 \beta 1$ ligand), and tenascin (an integrin $\alpha 8 \beta 1$ ligand) $(26,27,64)$, and excessive ECM deposition correlates with a high metastatic potential and the development of chemoresistance in SCLC (26). Integrin $\beta 1$ is considered the dominant type of $\beta$ integrin $(\beta 1>>\beta 2 \geqq \beta 3 \approx \beta 4$ ) detected in human SCLC cell lines (17). And another study using Northern blot analysis even indicated that ITGB1 is the only $\beta$ form of integrins detected in SCLC (23). These reports suggest that integrin $\beta 1$ might be the major integrin mediating the interaction between SCLC cells and the ECM. Besides the involvement in SCLC metastasis, integrin $\beta 1$ is also known to regulate chemoresistance (64). Previous studies on the posttranslational modulation of integrin $\beta 1$ indicate that several factors including c-Cbl, USP10, and SNX17 regulate integrin $\beta 1$ stability (65-67). Although none of these factors stood out in our screening of SCLC metastasis regulators, we identified the CUL5-SOCS3 E3 ligase complex as the master regulator responsible for integrin $\beta 1$ degradation and abundance control, at least in SCLC. These results are consistent with the significant association between the high integrin $\beta 1$ levels and the low CUL5 and SOCS3 levels observed in clinical samples. Although integrin $\beta 1$ plays a crucial role in CRL5-deficient SCLC metastasis, other substrates controlled by the CUL5-SOCS3 complex might also 
A

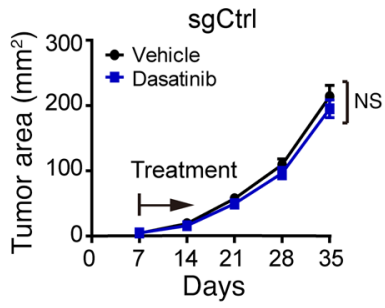

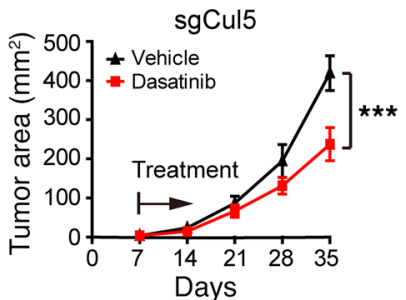

C
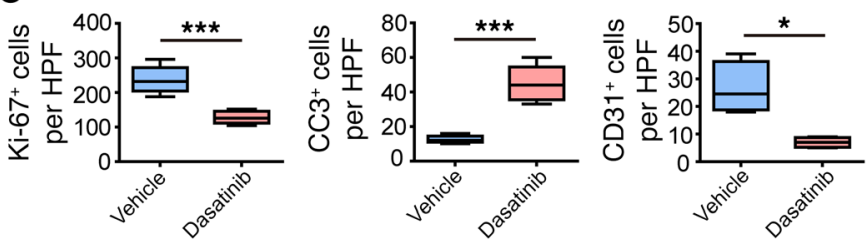

D

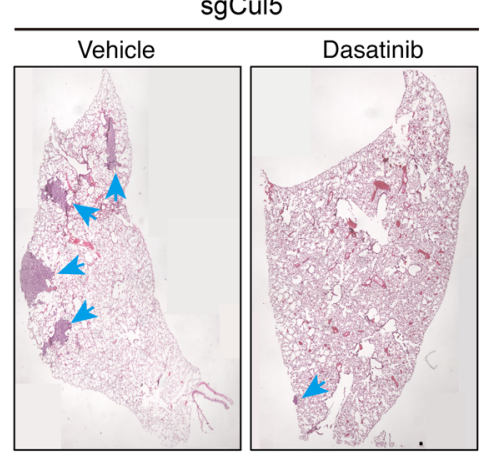

B

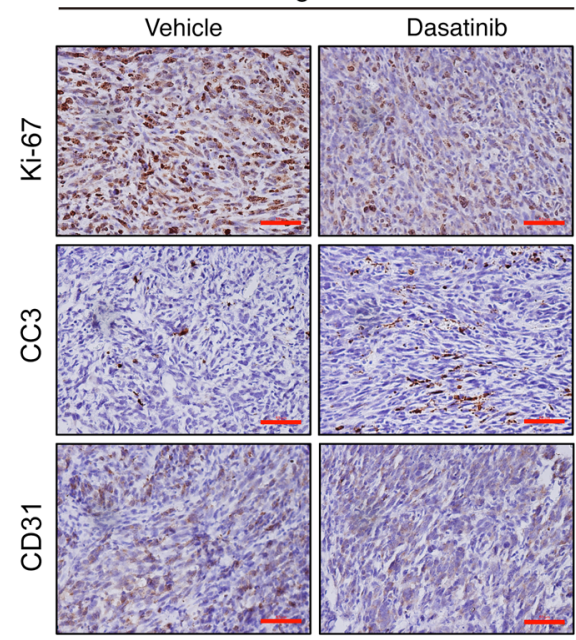

$\mathbf{F}$

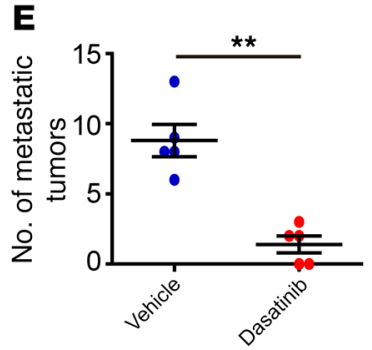

Figure 7. Treatment with the SRC inhibitor dasatinib significantly suppresses CUL5-defective mouse SCLC tumor growth and metastasis. (A) Dasatinib treatment in allograft assays with RT cells transduced with sgCtrl or sgCul5 ( $n=5$ mice per group). Data represent the mean \pm SEM. ${ }^{* * *} P<0.001$, by Student's $t$ test. (B) Immunohistochemical staining for Ki-67, CC3, and CD31, respectively, in samples from A. Scale bars: $50 \mu \mathrm{m}$. (C) Statistical analyses of the $\mathrm{Ki}-67^{+}, \mathrm{CC}^{+}$, and $\mathrm{CD} 31^{+}$index per HPF in allograft tumors of RT cells transduced with sgCul5. Data represent the mean \pm SEM. ${ }^{*} P<0.05$ and ${ }^{* * *} P<0.001$, by Student's $t$ test. (D) Representative H\&E staining of lung lobe sections from nude mice transplanted with RT cells transduced with sgCul5. Blue arrows indicate metastatic tumors. Scale bar: $50 \mu \mathrm{m}$. (E and F) Statistical analyses of metastatic tumor numbers and tumor burden from $\mathbf{D}$. Data represent the mean \pm SEM. ${ }^{*} P<0.05$ and ${ }^{* *} P<0.01$, by Student's $t$ test.

contribute to SCLC metastasis and will need to be further clarified in future studies.

Recent progress in basic cancer biology has helped the development of effective targeted therapy for many cancer types $(68$, 69). However, the therapeutic options for SCLC treatment are still largely limited. Understanding the mechanisms underlying the metastatic nature of SCLC is the key to improving the clinical treatment of SCLC. Kinases represent ideal targets for rational targeted therapies, given their well-established druggability (70, 71). We found that CUL5 and SOCS3 deficiency promoted SCLC cell migration and metastasis via integrin $\beta 1 / F A K / S R C$ pathways and that the depletion of FAK and SRC kinases strongly blocked the metastasis of CUL5- and SOCS3-deficient SCLC. Thus, inhibition of FAK/SRC signaling could potentially serve as an effective therapy for this specific subset of SCLC. Studies in multiple model systems support the high efficacy of the SRC inhibitor dasatinib in inhibiting metastasis $(56,72)$. Given these substantial preclinical observations, dasatinib appears promising for the treatment of metastatic tumors. Along this line of thought, we tested the efficacy of dasatinib in a tumor metastasis assay and found that CUL5-deficent SCLCs are vulnerable to dasatinib treatment. This indicates that dasatinib may be an effective drug for treating clini- cal SCLC with CUL5 and SOCS3 deficiency, in addition to its current application in leukemia treatment (73). It is also plausible that combining dasatinib with traditional chemotherapy such as etoposide might improve the clinical outcome of CUL5-defective SCLC. Taken together, we believe our study not only provides mechanistic insight into SCLC metastasis but also sheds new light on the potential for further improvements in the treatment of SCLC.

\section{Methods}

Reagents and antibodies. DMSO, cycloheximide (CHX), Tween-80, and polyethylene glycol (PEG-400) were purchased from MilliporeSigma. MLN4924 and MG132 were purchased from Selleck Chemicals. MLN4924 and MG132 were dissolved in DMSO. Dasatinib was purchased from MedChemExpress. RNAase, DMEM, RPMI-1640, FBS, penicillin, and streptomycin were purchased from Invitrogen (Thermo Fisher Scientific). Puromycin was purchased from Invitrogen (Thermo Fisher Scientific). PVDF membranes (Hybond-P) were purchased from GE Healthcare Life Sciences. Protein A/G agarose was purchased from Santa Cruz Biotechnology.

The following antibodies were used for immunoblotting: integrin $\beta 1$ (catalog ab52971) and integrin $\alpha 2$ (catalog ab181548) (both from Abcam); SRC pY416 (product no. 2101s), CC3 (product no. 9661s), Myc (product 
A

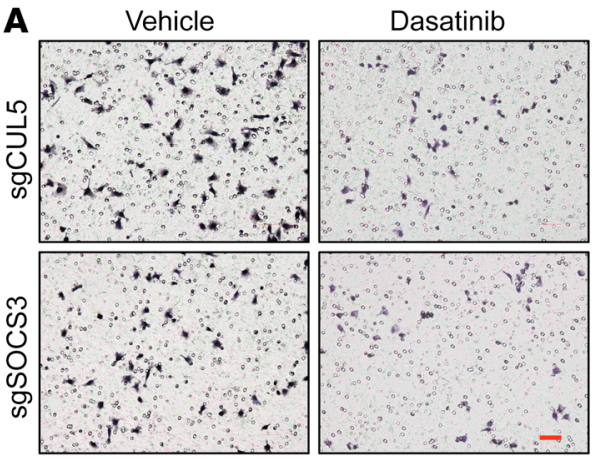

C sgCtrl

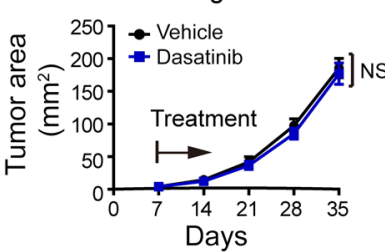

B

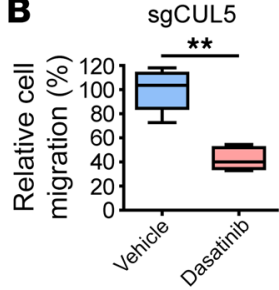

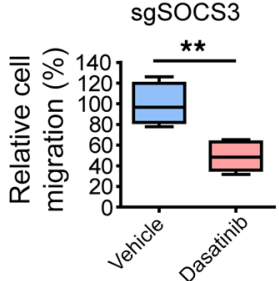

D

sgCUL5

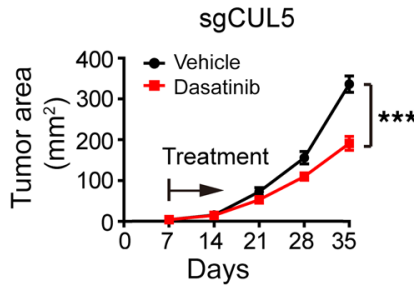

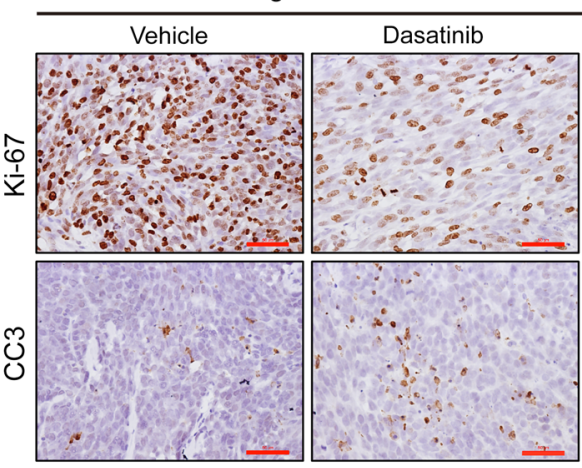

CUL5-SOCS3 deficiency

E
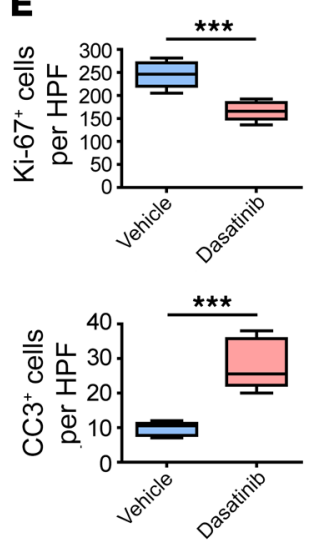

$\mathbf{F}$

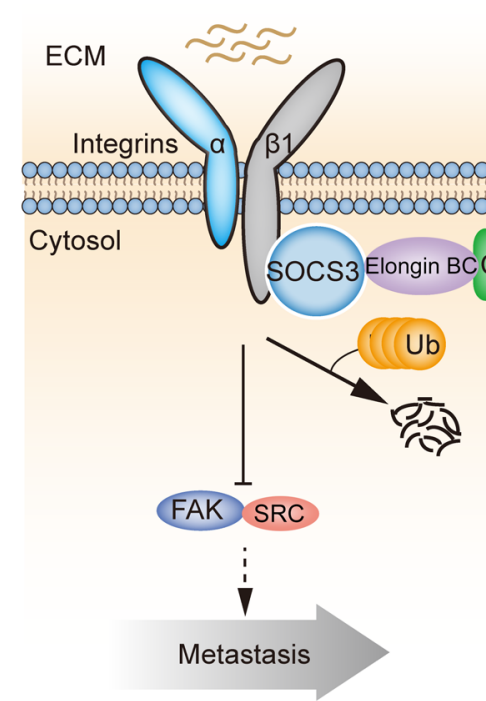

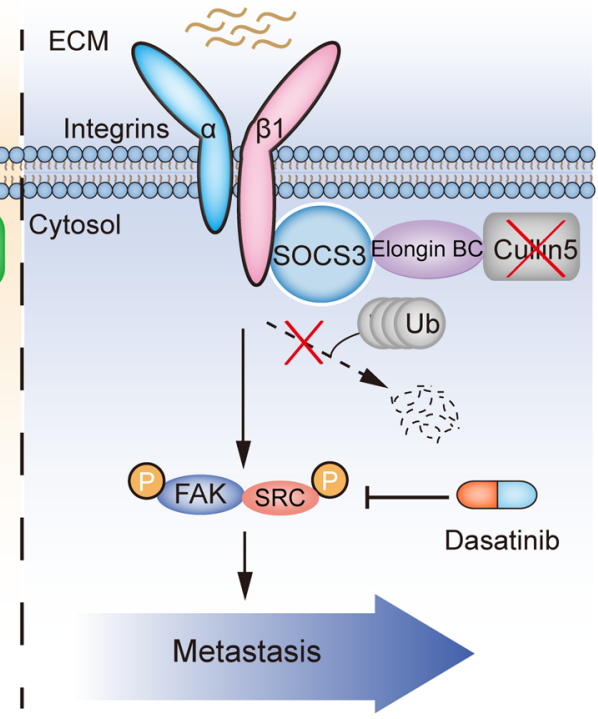

Figure 8. Dasatinib treatment results in the regression of CUL5-defective human SCLC tumors. (A) Representative images of migrated H345 cells transduced with sgCUL5 or sgSOCS3 and treated with $5 \mathrm{nM}$ dasatinib in a Transwell assay. Scale bar: $50 \mu \mathrm{m}$. (B) Quantification of the migrated cells in A. Data represent the mean \pm SEM. ${ }^{* *} P<0.01$, by Student's $t$ test. (C) Dasatinib treatment in xenograft assays with $\mathrm{H} 345$ cells transduced with sgCtrl or sgCUL5 ( $n=5$ mice per group). Data represent the mean \pm SEM. ${ }^{* * *} P<0.001$, by Student's $t$ test. (D) Immunohistochemical staining for Ki- 67 and CC3, respectively, in samples from C. Scale bar: $50 \mu \mathrm{m}$. (E) Statistical analyses of the Ki-67+ and CC $3^{+}$index per HPF in xenograft tumors of $\mathrm{H} 345$ cells transduced with sgCUL5. Data represent the mean \pm SEM. ${ }^{* *} P<0.001$, by Student's $t$ test. (F) Proposed model of CUL5-SOCS3 E3 ligase-mediated suppression of the integrin $\beta 1 / F A K / S R C$ prometastatic axis in SCLC. The CUL5-SOCS3 complex inhibits metastasis by promoting integrin $\beta 1$ ubiquitination and degradation, leading to decreased FAK/SRC signaling. Deficiency of the CUL5-SOCS3 complex impairs integrin $\beta 1$ ubiquitination and degradation, leading to integrin $\beta 1$ deposition and subsequent activation of FAK/SRC signaling and resulting in enhanced SCLC metastasis. The SRC inhibitor dasatinib treatment inhibits metastasis in CUL5- and SOCS3-defective SCLC.

no. 2278), p27 (product no. 3686), and TANK-binding kinase 1 (TBK1) (product no. 3504) (all from Cell Signaling Technology); FAK pY397 (product no. 04974) (from MilliporeSigma); GST (catalog 66001-1-AP) and SOCS3 (catalog 14025-1-AP) (both from Proteintech); FAK (catalog 610087) (from BD Transduction Laboratories); His (product no. H1029) and Flag (product no. F3165) (both from MilliporeSigma); tubulin, actin, and GAPDH (all from ABclonal); Cullin5 (catalog sc-373822), integrin $\alpha 1$ (catalog sc-271034), integrin $\beta 1$ (catalog sc-53711), SRC (catalog sc-8056), HA (catalog sc-7392), and HRP-conjugated rabbit and mouse secondary antibodies (all from Santa Cruz Biotechnology).

Cell culturing and drug treatment. The mouse primary SCLC cell line RT was established from de novo tumors from the $R b 1^{f / f l} \operatorname{Trp} 53^{f / f l}$ 
mouse model (6). The human SCLC cell line H345, the human cervical cancer cell line HeLa, and human embryonic kidney cells (HEK293T) were obtained from the American Type Culture Collection (ATCC). The H345 and RT cell lines were maintained in RPMI 1640 (Hyclone), while the HEK293T and HeLa cells were cultured in DMEM (Hyclone) supplemented with $10 \%$ FBS and subcultured every 2 days. Viral infection was performed as previously described (74). In all experiments, cells in the log phase were seeded for 24 hours, and the medium was replaced with fresh medium with or without drugs.

Genome-scale CRISPR/Cas9 screen. The mGeCKOa library was obtained from Addgene (catalog 1000000052). To produce virus, the mGeCKOa pooled plasmids were cotransfected into HEK293T cells with the lentiviral packaging plasmids psPAX2 and pMD2.G (Addgene plasmids 12260 and 12259). Briefly, HEK293T cells were seeded in $10-\mathrm{cm}$ dishes until about $80 \%$ confluence and then transfected with $8 \mu \mathrm{g}$ mGeCKOa, $6 \mu \mathrm{g}$ psPAX2, and $4 \mu \mathrm{g}$ pMD2.G, and the viral particles were harvested 48 hours after the change with fresh media. The mouse SCLC cell line RT was then infected with lentivirus carrying the mGeCKOa library or tomato control (sgCtrl) at a MOI of 0.3 , and selection was performed 48 hours later with $10 \mu \mathrm{g} / \mathrm{ml}$ puromycin. After in vitro culture for 1 week, a total of $6 \times 10^{7}$ cells were subcutaneously transplanted into the right side flank of nude mice $(n=60 ; 1 \times$ $10^{6}$ for each mouse) (Lingchang Company). The library representation (cells per lentiviral CRISPR construct) was greater than 400×. Seven weeks after transplantation, the mice were sacrificed and analyzed for metastatic tumors in distant organs including the lungs and liver. The metastasis tumors were extracted for genomic DNA and PCR amplified for the genomic region flanking sgRNA target sites, which were subjected to Sanger sequencing. Through the blast analyses in the NCBI database, the candidate genes were identified and are listed in Supplemental Table 1. The primers used for Sanger sequencing are listed in Supplemental Table 2.

Genomic editing with sgRNA. Genomic mutations in RT and H345 cells were generated using the CRISPR/Cas9 system as previously described $(43,75)$. sgRNA target sequences were designed using publicly available software (http://crispr.mit.edu/) and inserted into the lentiCRISPRv2 (Addgene, catalog 98290) or pSECC (Addgene, cata$\log 60820$ ) vector using the BsmBI restriction enzyme. SgRNA against the tomato gene was used as a negative control ( $\mathrm{sgCtrl}$ ). All the sgRNA target sequences used are listed in Supplemental Table 3.

shRNA constructs. Sequences for constructing shRNA targeting CUL5 or SOCS3 were obtained from MilliporeSigma and are listed in Supplemental Table 3. Various shRNAs against target genes were cloned into the pLKO.1 vector.

Plasmid constructs and mutagenesis. Human and mouse CUL5, SOCS3, or ITGB1 were PCR amplified and cloned into the pCDH-puro, pLEX-flag, pcDNA3.1, or pGEX-4T-1 vector. All primers used are listed in Supplemental Table 2. ITGB1-Flag construct was a gift of Jianfeng Chen (Shanghai Institute of Biochemistry and Cell Biology). ITGB1 or CUL5 point mutations were generated using the QuickChange Site-Directed Mutagenesis Kit (Stratagene).

Lentivirus production and infection. Lentiviral packaging and infection were done as previously described (74). Briefly, HEK293T cells were cotransfected with pCDH, pLEX, Lenti-vector, or pLKO.1 constructs and the packaging plasmids psPAX2 and pMD2.G. All media were removed after 6 to 8 hours and replaced with fresh DMEM plus $10 \%$ FBS. Viruses were collected and filtered with a $0.45-\mu \mathrm{m}$ mem- brane (Merck Millipore) 48 hours after replacement with fresh media. Virally infected cells were selected in puromycin for 96 hours.

Real-time semiquantitative PCR. Total RNA was extracted from cells using TRIzol Reagent (Invitrogen, Thermo Fisher Scientific) according to the manufacturer's instructions. Total RNA was retrotranscribed into first-strand cDNA using the RevertAid First Strand cDNA Synthesis Kit (Fermentas). The cDNAs were then used for regular PCR or real-time PCR (qPCR) on a 7500 Fast Real-Time PCR System (Applied Biosystems) using SYBR Green Master PCR Mix (Roche). Actin served as an internal control. The relative quantification of gene expression was analyzed by the $2^{-\Delta \Delta \mathrm{Ct}}$ method. The primers used for qPCR analyses are listed in Supplemental Table 2.

Co-IP. HEK293T cells were transfected with the plasmids using Lipofectamine LTX Reagent with PLUS Reagent (Invitrogen, Thermo Fisher Scientific) according to the manufacturer's instructions. Cells were lysed, and the supernatants were incubated with the antibodies and protein $\mathrm{A} / \mathrm{G}$ agarose. The incubation was performed at $4^{\circ} \mathrm{C}$ for 4 hours and then washed 4 times with NETN buffer (20 mM Tris-HCL [pH 8.0], $100 \mathrm{mM} \mathrm{NaCl}, 0.5 \%$ NP40, 1 mM EDTA). The immune complexes were subjected to SDS-PAGE and analyzed by immunoblotting.

GST-pulldown assay. Purification of GST-tag-fused recombinant proteins and GST-pulldown analyses were performed as described previously (76). Briefly, the pGEX4T1-SOCS3 plasmid was transformed into BL21-competent (DE3-competent) cells. The recombinant GST-SOCS3 proteins were expressed by isopropyl $\beta$-D-1-thiogalactopyranoside (IPTG) induction for 18 hours at $16^{\circ} \mathrm{C}$. The proteins were purified using GST agarose (GE Healthcare, 17-0756-01) according to the manufacturer's instructions. Agarose-bound GST-SOCS3 proteins were further incubated with eukaryotic protein Flag-integrin $\beta 1$. The incubation was performed at $4^{\circ} \mathrm{C}$ for 4 hours followed by washing 4 times with NETN buffer. Samples were resolved by SDS-PAGE and subjected to immunoblot (IB) analyses.

IB analyses. IB analyses were performed as described previously (77). Briefly, whole-cell lysates (WCLs) were prepared in lysis buffer supplemented with protease inhibitors (Roche). Equal amounts of protein were resolved by electrophoresis on gradient gels (Bio-Rad) and electrotransferred onto a PVDF membrane. After incubation in blocking buffer (50 mM Tris-buffered saline [pH 7.4] containing 5\% nonfat dry milk and $0.1 \%$ Tween-20), the membranes were probed with the primary antibodies, followed by incubation with HRP-conjugated goat anti-rabbit IgG or goat anti-mouse IgG. Bands were revealed with an ECL kit (Thermo Fisher Scientific) and recorded on x-ray films (Carestream). Images were captured using the FluorChem 8000 imaging system (Alpha Innotech).

Cell proliferation assay. For cell proliferation assays, 800 RT cells were seeded in 96-well plates (Nest), and the viability of the cells was measured at various time points. At specified time points (days 1-5), $20 \mu \mathrm{l}$ MTS (Promega) solution was added to each well, and the cells were incubated at $37^{\circ} \mathrm{C}$ for 2 hours. Next, absorbance was measured in single-wavelength mode (490-nm) using a Multiskan MK3 Microplate Reader (Thermo Fisher Scientific).

For human SCLC cell proliferation and cytotoxicity assays, 2000 cells were seeded in 96-well plates (Corning), and the viability of the cells was measured at various time points. At specified time points, $100 \mu$ CellTiter-Glo buffer (Promega) was added to each well. Next, absorbance was measured using a BioTek Synergy Neo Microplate Reader. 
Soft agar colony formation assay. Anchorage-independent cell growth was assessed by a soft agar assay as described before (78). In brief, $3 \%$ melting agarose was prepared and mixed with RPMI 1640 to make $0.4 \%$ and $1 \%$ agarose at $40^{\circ} \mathrm{C}$. Two milliliters of $1 \%$ agarose was added to the bottom of the six-well plate. Cells (6000 per well) were mixed with $2 \mathrm{ml}$ of $0.4 \%$ agarose, and the mixture was added on top of the $1 \%$ agarose. After routine culturing for 3 weeks, the cells were stained with $0.005 \%$ crystal violet, and the colonies $(>1 \mathrm{~mm}$ in diameter) were counted. All experiments were performed in triplicate.

Anoikis assay. Anoikis was induced by plating cells on polyHEMA-coated culture plates. Twenty-four hours after plating, the cells were collected by gentle pipetting and centrifuged to pellet down for the apoptosis assay.

Transwell migration assay. For cell migration, $1 \times 10^{5}$ cells were plated onto Transwell filters with 8-mm pores, a 24-well plate chamber insert (Corning) coated with Matrigel (Corning), and RPMI 1640 mixture (1:8). The top of the insert was supplemented with serum-free medium, while the bottom was supplemented with RPMI 1640 with $10 \%$ FBS. Cells were incubated for 24 hours and fixed with $4 \%$ paraformaldehyde for 15 minutes. After washing with PBS, cells at the top of the insert were scraped with a cotton swab. Cells adherent to the bottom were stained with hematoxylin for 1 minute and then washed 3 times with double-distilled $\mathrm{H}_{2} \mathrm{O}$. The positively stained cells on the underside of the filters were photographed and examined under the microscope.

Postsurgical metastasis model. The mouse SCLC RT cells with or without Cul5 knockout were subcutaneously injected into the flanks of nude mice. Primary tumor growth was monitored twice a week by caliper measurements. Tumor volume (V) was calculated with the following formula: $\mathrm{V}=\left(\mathrm{L} \times \mathrm{W}^{2}\right) / 2$, where $\mathrm{L}$ represents the larger diameter and $\mathrm{W}$ represents the smaller diameter. To remove primary tumors of comparable volume, the Cul5-knockout tumors were surgically removed on day 14, and the control tumors were removed on day 21. Four weeks later, the mice were sacrificed and analyzed for distant organ metastases.

CTC collection and analyses. Mouse SCLC RT cells stably expressing GFP fluorescence were sorted using a BD FACSAria II flow cytometer. The $\mathrm{GFP}^{+} \mathrm{RT}$ cells with or without Cul5 knockout were subcutaneously transplanted into the flanks of nude mice. Mice were anesthetized with $2.5 \%$ avertin, and a 5-mm-pore-diameter needle was used to puncture the orbit and aspirate $200 \mu \mathrm{l}$ whole blood into a 1-ml syringe primed with heparin sodium, and the blood was directly injected into a K2 EDTA-coated BD 367481 Vacutainer. Approximately $100 \mu \mathrm{l}$ whole blood was added to $5 \mathrm{ml}$ of $1 \times \mathrm{RBC}$ lysis buffer, pelleted, and resuspended in $5 \mathrm{ml}$ PBS with 0.5\% FBS. Cells were then pelleted and resuspended in $500 \mu \mathrm{lBS}$, and GFP positivity mirroring CTCs was measured on a BD LSR II flow cytometer.

Animal studies. Specific pathogen-free, 5-week-old male BALB/c nude mice were purchased from the Lingchang Company and housed under pathogen-free conditions in microisolation cages. The animals were acclimated for 1 week before experiments. The mouse or human SCLC cells $\left(1 \times 10^{6}\right.$ cells $/ 100 \mu$ in PBS $)$ in the logarithmic growth phase were subcutaneously injected into the right flank of nude mice. One week after inoculation, the mice were intragastrically administrated vehicle (5\% PEG-400 and 1\% Tween-80 in PBS) or dasatinib (30 mg/ $\mathrm{kg}$ ) for 4 consecutive weeks. The dosage of dasatinib used was based on previous reports (72) and our preliminary experiments. The tumor area (A) was measured with a caliper weekly and calculated with the formula: $\mathrm{A}=\mathrm{L} \times \mathrm{W}$. At various time points, the mice were sacrificed for further molecular and pathological analyses.

$R b 1^{f / f l} \operatorname{Trp} 53^{f / f l}$ mouse model studies. Conditional Rb1 $1^{f / f l} \operatorname{Trp} 53^{f / / f l}$ (RT) mice were maintained on a mixed genetic background (C57Bl/6, $\mathrm{BALB} / \mathrm{c}$, and S129) as previously described (79). Mice at 8 weeks of age were given adenovirus-CMV-Cre recombinase (Adeno-Cre, $2 \times$ $10^{6} \mathrm{PFU}$ ) by intratracheal intubation (80) to allow for Cre-lox-mediated recombination of floxed $\operatorname{Trp} 53$ and $R b 1$ alleles. Mice were then aged up to 32 weeks to allow for SCLC disease development. Primary lung tumors and/or metastatic liver tumors freshly dissected from RT mice $(n=10) 32$ weeks after Adeno-Cre infection were all included for pathological analyses including H\&E and IHC staining.

$H \& E$ and immunohistochemical staining. $\mathrm{H} \& \mathrm{E}$ and immunohistochemical stainings were performed as previously described $(78,81)$. Briefly, mice were sacrificed and lung tissues were inflated and fixed in $10 \%$ formalin, embedded in paraffin, sectioned at $5-\mu \mathrm{m}$ thickness, and then stained with H\&E. Tumor numbers and burden were measured in 5 continuous sections with $100-\mu \mathrm{M}$ intervals in each mouse.

For immunohistochemical staining, slides were deparaffinized in xylene and ethanol and rehydrated in water. Antigen retrieval was performed by heating slides in a microwave for 20 minutes in sodium citrate buffer ( $\mathrm{pH}$ 6.0). Slides were quenched in hydrogen peroxide (3\%) to block endogenous peroxidase activity and then washed in TBS with Tween-20 (TBST) buffer. The primary antibodies were incubated at $4^{\circ} \mathrm{C}$ overnight followed by use of the SuperPicture Polymer Detection Kit (Life Technologies, Thermo Fisher Scientific) according to the manufacturer's instructions and as previously described $(77,81)$. To calculate the percentage of cells positive for Ki-67, CC3, or CD31 in the immunohistochemical stainings, at least 10 to 15 high-power fields (HPF) from each mouse and a total of over $100 \mathrm{HPF}$ were randomly selected for statistical analyses as described previously $(74,77)$. The following antibodies were used for immunostaining: integrin $\beta 1$ (catalog ab52971, Abcam); CC3 (product no. 9661s, Cell Signaling Technology); SOCS3 (catalog 14025-1-AP, ProteinTech); Ki-67 (product no. NCL-Ki67p, Leica Biosystems); CD31 (product no. D160721, Sangon); CUL5 (catalog sc-373822, Santa Cruz Biotechnology); and NCAM (catalog A7913, ABclonal).

Human lung cancer specimens. Human SCLC tissue specimens were used for immunohistochemical staining for CUL5, SOCS3, and integrin $\beta 1$ and statistical analyses. Immunohistochemical staining was blindly scored as low or high according to staining density and subjected to analysis for clinical relevance as previously described $(74,77)$.

Statistics. Statistical analysis was performed using GraphPad Prism 5 (GraphPad Software). Unless otherwise indicated, data were analyzed by 2 -tailed Student's $t$ test for 2 groups, and 1-way ANOVA followed by the Newman-Keuls multiple comparisons test was used to analyze statistical significance among multiple groups. Survival analysis was performed using the Kaplan-Meier method and comparisons made by logrank (Mantel-Cox) test. Data are shown as the mean \pm SEM. A $P$ value of less than 0.05 was considered statistically significant.

Study approval. Animal experiments were conducted following the NIH guidelines and were approved by the IACUC of the Institute of Biochemistry and Cell Biology, Shanghai Institutes for Biological Sciences, Chinese Academy of Sciences. Human SCLC specimens were collected with the approval of the institutional review committee of Zhejiang Cancer Hospital (Hangzhou, China). All patients gave written informed consent. 


\section{Author contributions}

HJ, DG, GZ, and LG conceived the study and designed the experiments. GZ and LG performed most experiments and analyzed the data. YJ, CG, MY, SY, ZQ, YY, YT, QW, JZ, BC, QD, HH, LH, YC, PZ, GH, LC, and KKW provided materials and technical assistance. DS provided human SCLC specimens. HJ, DG, GZ, and LG wrote the manuscript.

\section{Acknowledgments}

We are very grateful to the patients and their families. We thank Ronald Depinho for providing the $R b 1^{f / f l} \operatorname{Tr} p 53^{f / f l}$ mouse model and T. Jacks, W. Xue, and F.J. Sanchez-Rivera for providing the pSECC and LentiCRISPRv2 plasmids. We are grateful to Fei Li, Zhaoyuan Fang, Shiyu Zhou, and Dan Sun (all from Shanghai Institute of Biochemistry and Cell Biology, Chinese Academy of Sciences) for helpful comments and technical assistance. This work was supported by the National Basic Research Program of
China (grants 2017YFA0505501 and 2015CB964502); the Strategic Priority Research Program of the Chinese Academy of Sciences (grants XDB19020201 and XDB19020203); the National Natural Science Foundation of China (grants 81430066, 91731314, 81790253, 81872312, 81871875, 81802279, and 31621003); the Science and Technology Commission of Shanghai Municipality (grant 15XD1504000); and the China Postdoctoral Science Foundation (2016M601667).

Address correspondence to: Hongbin Ji or Daming Gao, State Key Laboratory of Cell Biology, Innovation Center for Cell Signaling Network, CAS Center for Excellence in Molecular Cell Science, Shanghai Institute of Biochemistry and Cell Biology, Chinese Academy of Sciences; University of Chinese Academy of Sciences, 320 Yue Yang Road, Shanghai, 200031, China. Phone: 86.21.54921107; Email: hbji@sibcb.ac.cn (HJ). Phone: 86.21.54921292; Email: dgao@sibcb.ac.cn (DG).
1. van Meerbeeck JP, Fennell DA, De Ruysscher DK. Small-cell lung cancer. Lancet. 2011;378(9804):1741-1755.

2. Früh M, et al. Small-cell lung cancer (SCLC): ESMO Clinical Practice Guidelines for diagnosis, treatment and follow-up. Ann Oncol. 2013;24(Suppl 6):vi99-vi105.

3. Jänne PA, et al. Twenty-five years of clinical research for patients with limited-stage small cell lung carcinoma in North America. Cancer. 2002;95(7):1528-1538.

4. Kalemkerian GP, et al. Small cell lung cancer. J Natl Compr Canc Netw. 2013;11(1):78-98.

5. George J, et al. Comprehensive genomic profiles of small cell lung cancer. Nature. 2015;524(7563):47-53.

6. Meuwissen R, Linn SC, Linnoila RI, Zevenhoven J, Mooi WJ, Berns A. Induction of small cell lung cancer by somatic inactivation of both Trp53 and $\mathrm{Rb} 1$ in a conditional mouse model. Cancer Cell. 2003;4(3):181-189.

7. Calbo J, et al. A functional role for tumor cell heterogeneity in a mouse model of small cell lung cancer. Cancer Cell. 2011;19(2):244-256.

8. Denny SK, et al. Nfib promotes metastasis through a widespread increase in chromatin accessibility. Cell. 2016;166(2):328-342.

9. Gardner EE, et al. Chemosensitive relapse in small cell lung cancer proceeds through an EZH2SLFN11 axis. Cancer Cell. 2017;31(2):286-299.

10. Rudin CM, et al. Comprehensive genomic analysis identifies SOX2 as a frequently amplified gene in small-cell lung cancer. Nat Genet. 2012;44(10):1111-1116.

11. Mollaoglu G, et al. MYC drives progression of small cell lung cancer to a variant neuroendocrine subtype with vulnerability to aurora kinase inhibition. Cancer Cell. 2017;31(2):270-285.

12. Nau MM, et al. L-myc, a new myc-related gene amplified and expressed in human small cell lung cancer. Nature. 1985;318(6041):69-73.

13. Takebe N, et al. Targeting Notch, Hedgehog, and Wnt pathways in cancer stem cells: clinical update. Nat Rev Clin Oncol. 2015;12(8):445-464.

14. Lim JS, et al. Intratumoural heterogeneity generated by Notch signalling promotes small-cell lung cancer. Nature. 2017;545(7654):360-364.

15. Johnson BE, et al. Changes in the phenotype of human small cell lung cancer cell lines after transfection and expression of the c-myc proto-oncogene. J Clin Invest. 1986;78(2):525-532.

16. Johnson BE, et al. Restriction fragment length polymorphism studies show consistent loss of chromosome $3 p$ alleles in small cell lung cancer patients' tumors. J Clin Invest. 1988;82(2):502-507.

17. Feldman LE, Shin KC, Natale RB, Todd RF. Beta 1 integrin expression on human small cell lung cancer cells. Cancer Res. 1991;51(4):1065-1070.

18. Oshita F, et al. High expression of integrin beta1 and $\mathrm{p} 53$ is a greater poor prognostic factor than clinical stage in small-cell lung cancer. Am JClin Oncol. 2004;27(3):215-219.

19. Hynes RO. Integrins: versatility, modulation, and signaling in cell adhesion. Cell. 1992;69(1):11-25.

20. Desgrosellier JS, Cheresh DA. Integrins in cancer: biological implications and therapeutic opportunities. Nat Rev Cancer. 2010;10(1):9-22.

21. Hynes RO. Integrins: bidirectional, allosteric signaling machines. Cell. 2002;110(6):673-687.

22. Schlaepfer DD, Hauck CR, Sieg DJ. Signaling through focal adhesion kinase. Prog Biophys Mol Biol. 1999;71(3-4):435-478.

23. Falcioni R, et al. Expression of beta 1, beta 3, beta 4 , and beta 5 integrins by human lung carcinoma cells of different histotypes. Exp Cell Res. 1994;210(1):113-122.

24. Lawson $\mathrm{MH}$, et al. Bcl-2 and $\beta 1$-integrin predict survival in a tissue microarray of small cell lung cancer. Br J Cancer. 2010;103(11):1710-1715.

25. Oshita F, et al. Increased expression of integrin beta1 is a poor prognostic factor in small-cell lung cancer. Anticancer Res. 2002;22(2B):1065-1070.

26. Buttery RC, Rintoul RC, Sethi T. Small cell lung cancer: the importance of the extracellular matrix. Int J Biochem Cell Biol. 2004;36(7):1154-1160.

27. Rintoul RC, Sethi T. The role of extracellular matrix in small-cell lung cancer. Lancet Oncol. 2001;2(7):437-442.

28. Petroski MD, Deshaies RJ. Function and regulation of cullin-RING ubiquitin ligases. Nat Rev $\mathrm{Mol}$ Cell Biol. 2005;6(1):9-20.
29. Weathington NM, Mallampalli RK. Emerging therapies targeting the ubiquitin proteasome system in cancer. JClin Invest. 2014;124(1):6-12.

30. Hochstrasser M. Lingering mysteries of ubiquitin-chain assembly. Cell. 2006;124(1):27-34

31. Huang DT, et al. E2-RING expansion of the NEDD8 cascade confers specificity to cullin modification. Mol Cell. 2009;33(4):483-495

32. Popov N, Schülein C, Jaenicke LA, Eilers M. Ubiquitylation of the amino terminus of Myc by SCF ( $\beta$-TrCP) antagonizes SCF(Fbw7)-mediated turnover. Nat Cell Biol. 2010;12(10):973-981.

33. Zheng N, et al. Structure of the Cul1-Rbx1-Skp1-F boxSkp2 SCF ubiquitin ligase complex. Nature. 2002;416(6882):703-709.

34. Latres E, Chiaur DS, Pagano M. The human F box protein beta-Trcp associates with the Cul1/Skp1 complex and regulates the stability of betacatenin. Oncogene. 1999;18(4):849-854.

35. Mahrour N, et al. Characterization of Cullin-box sequences that direct recruitment of Cul2-Rbx1 and Cul5-Rbx2 modules to Elongin BC-based ubiquitin ligases. J Biol Chem. 2008;283(12):8005-8013.

36. Babon JJ, Sabo JK, Zhang JG, Nicola NA, Norton RS. The SOCS box encodes a hierarchy of affinities for Cullin5: implications for ubiquitin ligase formation and cytokine signalling suppression. JMol Biol. 2009;387(1):162-174.

37. Hilton DJ, et al. Twenty proteins containing a C-terminal SOCS box form five structural classes. Proc Natl Acad Sci U S A. 1998;95(1):114-119.

38. Kile BT, Schulman BA, Alexander WS, Nicola NA, Martin HM, Hilton DJ. The SOCS box: a tale of destruction and degradation. Trends Biochem Sci. 2002;27(5):235-241.

39. Zhou W, et al. Neddylation E2 UBE2F promotes the survival of lung cancer cells by activating CRL5 to degrade NOXA via the K11 linkage. Clin Cancer Res. 2017;23(4):1104-1116.

40. Teckchandani A, et al. Cullin 5 destabilizes Cas to inhibit Src-dependent cell transformation. J Cell Sci. 2014;127(Pt 3):509-520.

41. Liu D, et al. SOCS3 drives proteasomal degradation of TBK1 and negatively regulates antiviral innate immunity. Mol Cell Biol. 
2015;35(14):2400-2413.

42. Chen S, et al. Genome-wide CRISPR screen in a mouse model of tumor growth and metastasis. Cell. 2015;160(6):1246-1260.

43. Sanjana NE, Shalem O, Zhang F. Improved vectors and genome-wide libraries for CRISPR screening. Nat Methods. 2014;11(8):783-784.

44. Petrilli AM, Fernández-Valle C. Role of Merlin/ NF2 inactivation in tumor biology. Oncogene. 2016;35(5):537-548.

45. Kamura T, et al. VHL-box and SOCS-box domains determine binding specificity for Cul2Rbx1 and Cul5-Rbx2 modules of ubiquitin ligases. Genes Dev. 2004;18(24):3055-3065.

46. Babon JJ, et al. The SOCS box domain of SOCS3: structure and interaction with the elonginBC-cullin5 ubiquitin ligase. J Mol Biol. 2008;381(4):928-940.

47. Wieland E, et al. Endothelial Notch1 activity facilitates metastasis. Cancer Cell. 2017;31(3):355-367.

48. Krebs MG, Metcalf RL, Carter L, Brady G, Blackhall FH, Dive C. Molecular analysis of circulating tumour cells-biology and biomarkers. Nat Rev Clin Oncol. 2014;11(3):129-144

49. Soucy TA, et al. An inhibitor of NEDD8-activating enzyme as a new approach to treat cancer. Nature. 2009;458(7239):732-736.

50. Komander D, Rape M. The ubiquitin code. Annu Rev Biochem. 2012;81:203-229.

51. Orabona C, et al. SOCS3 drives proteasomal degradation of indoleamine 2,3-dioxygenase (IDO) and antagonizes IDO-dependent tolerogenesis. Proc NatlAcad Sci US A. 2008;105(52):20828-20833.

52. Zhang JG, et al. The conserved SOCS box motif in suppressors of cytokine signaling binds to elongins $B$ and $C$ and may couple bound proteins to proteasomal degradation. Proc Natl Acad Sci U S A. 1999;96(5):2071-2076.

53. Giancotti FG, Ruoslahti E. Integrin signaling. Science. 1999;285(5430):1028-1032.

54. Sieg DJ, Hauck CR, Schlaepfer DD. Required role of focal adhesion kinase (FAK) for integrinstimulated cell migration. JCell Sci. 1999;112(Pt 16):2677-2691.

55. Mitra SK, Schlaepfer DD. Integrin-regulated FAK-Src signaling in normal and cancer cells.
Curr Opin Cell Biol. 2006;18(5):516-523.

56. Ishizawar R, Parsons SJ. c-Src and cooperating partners in human cancer. Cancer Cell. 2004;6(3):209-214.

57. Avraamides CJ, Garmy-Susini B, Varner JA. Integrins in angiogenesis and lymphangiogenesis. $\mathrm{Nat}$ Rev Cancer. 2008;8(8):604-617.

58. Lang R, et al. SOCS3 regulates the plasticity of gp130 signaling. Nat Immunol. 2003;4(6):546-550.

59. Teckchandani A, Cooper JA. The ubiquitinproteasome system regulates focal adhesions at the leading edge of migrating cells. Elife. 2016;5:e17440.

60. Ogata $\mathrm{H}$, et al. Deletion of the SOCS3 gene in liver parenchymal cells promotes hepatitisinduced hepatocarcinogenesis. Gastroenterology. 2006;131(1):179-193.

61. Weber A, et al. SOCS-3 is frequently methylated in head and neck squamous cell carcinoma and its precursor lesions and causes growth inhibition. Oncogene. 2005;24(44):6699-6708.

62. He B, et al. SOCS-3 is frequently silenced by hypermethylation and suppresses cell growth in human lung cancer. Proc Natl Acad Sci U S A. 2003;100(24):14133-14138.

63. Friedl P, Wolf K. Tumour-cell invasion and migration: diversity and escape mechanisms. Nat Rev Cancer. 2003;3(5):362-374.

64. Sethi T, et al. Extracellular matrix proteins protect small cell lung cancer cells against apoptosis: a mechanism for small cell lung cancer growth and drug resistance in vivo. Nat Med. 1999;5(6):662-668.

65. Yang N, Yu F, Shao G, Fu Y, Kong W. The E3 ubiquitin ligase $\mathrm{c}-\mathrm{Cbl}$ mediates integrin $\beta 1$ ubiquitination during dilated cardiomyopathy. Biochem Biophys Res Commun. 2016;479(4):728-735.

66. Gillespie SR, Tedesco LJ, Wang L, Bernstein AM. The deubiquitylase USP10 regulates integrin $\beta 1$ and $\beta 5$ and fibrotic wound healing. J Cell Sci. 2017;130(20):3481-3495.

67. Steinberg F, Heesom KJ, Bass MD, Cullen PJ. SNX17 protects integrins from degradation by sorting between lysosomal and recycling pathways. JCell Biol. 2012;197(2):219-230.

68. Subbiah V, et al. Precision targeted therapy with
BLU-667 for RET-driven cancers. Cancer Discov. 2018;8(7):836-849.

69. Rotow J, Bivona TG. Understanding and targeting resistance mechanisms in NSCLC. Nat Rev Cancer. 2017;17(11):637-658.

70. Davis MI, et al. Comprehensive analysis of kinase inhibitor selectivity. Nat Biotechnol. 2011;29(11):1046-1051.

71. Ferguson FM, Gray NS. Kinase inhibitors: the road ahead. Nat Rev Drug Discov. 2018;17(5):353-377.

72. Desgrosellier JS, et al. An integrin alpha(v) beta(3)-c-Src oncogenic unit promotes anchorage-independence and tumor progression. Nat Med. 2009;15(10):1163-1169.

73. Gnoni A, Marech I, Silvestris N, Vacca A, Lorusso V. Dasatinib: an anti-tumour agent via Src inhibition. Curr Drug Targets. 2011;12(4):563-578.

74. Li F, et al. LKB1 Inactivation elicits a redox imbalance to modulate non-small cell lung cancer plasticity and therapeutic response. Cancer Cell. 2015;27(5):698-711.

75. Cong L, et al. Multiplex genome engineering using CRISPR/Cas systems. Science. 2013;339(6121):819-823.

76. Xie X, et al. The mTOR-S6K pathway links growth signalling to DNA damage response by targeting RNF168. Nat Cell Biol. 2018;20(3):320-331.

77. Gao Y, et al. LKB1 inhibits lung cancer progression through lysyl oxidase and extracellular matrix remodeling. Proc Natl Acad Sci U S A. 2010;107(44):18892-18897.

78. Ji H, et al. LKB1 modulates lung cancer differentiation and metastasis. Nature. 2007;448(7155):807-810

79. Jonkers J, Meuwissen R, van der Gulden $\mathrm{H}$, Peterse H, van der Valk M, Berns A. Synergistic tumor suppressor activity of BRCA2 and p53 in a conditional mouse model for breast cancer. Nat Genet. 2001;29(4):418-425.

80. DuPage M, Dooley AL, Jacks T. Conditional mouse lung cancer models using adenoviral or lentiviral delivery of Cre recombinase. Nat Protoc. 2009;4(7):1064-1072.

81. Han X, et al. Transdifferentiation of lung adenocarcinoma in mice with Lkb1 deficiency to squamous cell carcinoma. Nat Commun. 2014;5:3261. 